\title{
Adaptive rational equilibrium with forward looking agents
}

\author{
William Brock, ${ }^{*}$ Pietro Dindo ${ }^{\dagger}$ and Cars Hommes ${ }^{\ddagger}$
}

In adaptive rational equilibrium dynamics (ARED) agents choose between a costly rational expectation forecast and a cheap naive forecast, and the fractions using each of the two strategies evolve over time and are endogenously coupled to the market equilibrium price dynamics. In this setting, agents are backward looking in the sense that strategy selection is based on experience measured by relative past realized profits. When the selection pressure to switch to the more profitable strategy is high, instability and complicated chaotic price fluctuations arise.

In this paper we investigate the ARED with forward looking agents, whose strategy selection is based upon expected profits. Our findings suggest that forward looking behavior dampens the amplitude of price fluctuations, but local instability of the steady state remains. The global dynamics depends upon how sophisticated the forward looking behavior is. With perfectly forward looking agents, prices converge to a stable 2-cycle, whereas with forward looking agents who are boundedly rational concerning their estimate of expected profits, small amplitude chaotic price fluctuations might arise.

We also establish an equivalence relationship between a heterogeneous agent model with switching of strategies and a representative agent framework, where the representative agent optimally chooses between the benefits of a high quality forecast and the associated information gathering costs. To an outside observer it is impossible to distinguish between the two.

Key words heterogeneous beliefs, discrete choice dynamics, cobweb model, endogenous business cycles, chaos

JEL classification C60, D83, D84, E32

Accepted 7 April 2006

\footnotetext{
*Department of Economics, University of Wisconsin, Madison, WI, USA.

${ }^{\dagger}$ CeNDEF, Department of Quantitative Economics, University of Amsterdam, Amsterdam, the Netherlands.

${ }^{\ddagger}$ CeNDEF, Department of Quantitative Economics, University of Amsterdam, Amsterdam, the Netherlands. Email: C.H.Hommes@uva.nl

An earlier version of this paper was presented at the conference "Intertemporal Equilibria, Aggregation and Sunspots" organized in honor of Jean Michel Grandmont, Lisbon, 30-31 October 2005. Comments from conference participants and an anonymous referee are gratefully acknowledged.
} 


\section{Introduction}

The work of Jean-MichelGrandmont (e.g. Grandmont 1982, 1985, 1998) has played a most stimulating role in the debate whether adaptive learning might or might not lead to coordination and convergence to self-fulfilling expectations in economic dynamic systems. This debate is important, because instability of adaptive learning might explain why financial markets exhibit excess volatility; that is, are more volatile than justified by underlying economic fundamentals.

Grandmont (1982) surveys the field of temporary general equilibrium theory. In this theory, at each date $t$, agents form expectation functions for relevant quantities at date $t+1$, and given these expectations, agents optimize to produce demand functions at date $t$. Markets then clear at date $t$ to produce equilibrium prices and quantities at date $t$. At date $t+1$ the process is repeated for going into date $t+2$. A sequence of prices, quantities, and expectations, is produced. In principle, there could be feedback from equilibrium prices and quantities into expectations functions but the expectations need not be rational. At each date $t$, the actions of the agents are coordinated only by the price system. Unlike rational expectations the plans for the future that are made by the agents are not coordinated. As Grandmont (1982, p. 887) puts it:

The aim of temporary equilibrium theory is to study the interaction through markets of different individuals in a given period, and to analyze the behavior over time of the sequence of these equilibria.

Grandmont treats both spot markets and futures markets in his review and focuses mostly on cases where agents plan one period ahead. Radner (1982, p. 950) gives an early treatment of the rational expectations approach to general equilibrium and links it to the temporary general equilibrium approach.

More recently, Grandmont (1998) focuses on dynamics rather than conditions for existence in a general temporary equilibrium setting. Grandmont (1998, p. 743) identifies an "uncertainty principle" under which learning generates instability:

learning generates local instability of self-fulfilling expectations whenever agents are on average uncertain about the local dynamics of the system, and thus ready to extrapolate a wide range of regularities (trends) out of past deviations from equilibrium, and when the influence of expectations on the dynamics of the system is significant.

Moreover, on local instability and global dynamics Grandmont (1998, p. 743) notes:

I should emphasize that the instability we are talking about is only local, and that plausible global nonlinearities, originating from the agents' expectations scheme themselves, may keep the motion of the system bounded. Thus even in the absence of shocks to the "fundamentals", or to expectations, learning by itself might generate convergence to complex nonlinear ("chaotic") attractors, hence self perpetuating endogenous fluctuations, along which forecasting errors would never vanish.

Finally, Grandmont (1998, p. 744) puts discipline on such complex "learning equilibria": 
The ultimate test that this approach will have to pass, however, is that such "learning equilibria" must, to be acceptable, exhibit a reasonable degree of consistency with the agents' beliefs. In this respect, one might envision situations in which agents think that they are living in a world that is relatively simple, although subject to random shocks, but in which deterministic "learning equilibria" are complex (chaotic) enough to make the agents' forecasting mistakes still "self-fulfilling" in a well defined sense.

The purpose of our paper is to study a "toy model" of a compromise economy, along the lines of Brock and Hommes (1997), where it is costly to possess fully structurally rational expectations but less costly or free to possess "simpler" expectations. Our key objective is to study the dynamics of such an economy in the simplest set of minimalist models in which we can expose the ebb and flow of forces to possess fully structural rational expectations. Hence, if it were very costly to purchase fully structurally rational expectations our economy would act more like a Grandmont temporary general equilibrium economy. Instead, if fully structural rational expectations were cheap our economy would act more like a rational expectations economy. Although it would be an excellent research project to study such an economy with endogenous rational choice of the "level of rationality" in a general equilibrium system at the level of generality of Grandmont (1982) and Radner (1982), it turns out to be challenging enough to study the impact of this extra level of dynamics of information choice in a simple cobweb economy as we shall do here.

Brock and Hommes (1997) introduce the concept of adaptive rational equilibrium dynamics (ARED), which is an endogenous coupling between the selection of expectations rules and market equilibrium dynamics. In the ARED, the consistency requirement that has to be imposed upon learning rules, as emphasized by Grandmont, is an evolutionary selection of strategies; that is, agents switch to rules that have performed well in the recent past. ${ }^{1}$ Brock and Hommes (1998) apply this evolutionary switching mechanism to an asset pricing model with heterogeneous beliefs; see Hommes (2006) for an extensive survey of heterogeneous agent models in economics and finance.

As a simple illustration of the ARED concept, Brock and Hommes (1997) consider a cobweb model where agents can choose between two predictors: either a cheap naive predictor, where the forecast for the price for tomorrow is the last observed price, or an expensive rational expectation (perfect foresight) predictor. To choose between the two predictors, agents compare their performance as measured by relative past realized profit. One of the main results in Brock and Hommes (1997) is that, when the selection pressure to switch between a costly sophisticated rational and cheap simple naive strategy is high, the price dynamics becomes locally unstable. As prices diverge, errors from the simple, naive strategy increase and it becomes worthwhile to switch to the costly rational strategy, pushing prices back close to the steady state. This interaction between a "close to the steady state destabilizing force" and a "far from the steady state stabilizing force" leads

\footnotetext{
${ }^{1}$ Another type of complicated "learning equilibrium" in which forecasting mistakes are "self-fulfilling" in the spirit of Grandmont is the consistent expectations equilibrium (CEE), introduced in Hommes and Sorger (1998). In a CEE, agents use a simple linear forecasting rule in an unknown nonlinear economy. A CEE arises when the sample average and sample autocorrelations of the nonlinear implied law of motion coincide with the corresponding linear belief. One possibility is chaotic CEE with chaotic price fluctuations with sample average and sample autocorrelations exactly corresponding to a stochastic AR(1) process. See Bullard (1994), Schönhofer (1999) and Tuinstra (2003) for similar complicated learning equilibria.
} 
to complicated, chaotic price fluctuations. These complicated "learning equilibria" are driven by past realized net profits of the expectation strategies. More precisely, in the Brock and Hommes (1997) model strategy selection is given by a discrete choice model with a performance or fitness measure based upon past payoffs, (i.e. realized profits) from earlier choices of predictors. Therefore, strategy selection is based on "experience" or "regret", and agents tend to switch to strategies that have performed well in the (recent) past. In the Brock and Hommes (1997) model both the naive and the rational agents are backward looking with respect to the choice of their prediction strategy but forward looking in their production decision because they want to maximize expected profits. In fact, these agents implicitly use past profits as a proxy for expected future profits. One might argue that sophisticated agents should realize that other agents are making their choices in this backward looking manner and will learn to use a more sophisticated predictor selection strategy attempting to exploit the backward looking behavior. This reasoning raises the obvious question: Will the Brock and Hommes (1997) instability results vanish under a concept of sophisticated forward looking predictor strategy selection?

In this paper, we investigate ARED with agents who are forward looking concerning their selection of strategies. For this purpose we reconsider the same cobweb model as in Brock and Hommes (1997), in the case where agents evaluate the different predictors based upon their expected profit instead of their realized profits. As one might expect with hindsight, forward looking behavior does dampen some of the instabilities uncovered by the original Brock and Hommes (1997) work. The tendency to overshoot when "selection pressure" is high is dampened. Indeed, in one case the erratic dynamics is dampened down to a stable 2-cycle. In other cases, however, small amplitude chaotic price fluctuations persist.

Our setup is also related to the concept of quantal response equilibrium introduced by McKelvey and Palfrey $(1995,1998)$. They also use a discrete choice model for strategy selection in a game theoretic setting, with expected payoff as the performance measure. A similar approach is used by Camerer et al. (2002), who study repeated games with various levels of rationality. An important difference between these game theoretic settings and Brock and Hommes (1997) is that in the latter case strategy selection is coupled to the dynamics of an endogenous variable, say the market price, whose realization affects the performance of all strategies.

A second contribution of this paper is to formulate a representative agent version of the model, where the costs of information gathering of more sophisticated strategies are endogenized. This approach is inspired by Simon $(1955,1957)$ and more recently by Evans and Ramey (1992) and especially by Dudek (2004). We formulate hybrid models based on Brock and Hommes (1997) and Dudek (2004), where a representative agent chooses optimally among predictors of different quality, where quality is purchased at a cost that is increasing and convex in quality. We establish a close link between the representative agent optimizing between the benefits of sophisticated prediction rules and information gathering costs and a heterogeneous agent framework with switching of strategies. See Kirman (1992) for a critique upon the representative agent approach in economics and Hommes (2006) and LeBaron (2006)for surveys of heterogeneous agent modeling. 
The paper is organized as follows. Section 2 reviews some facts from Brock and Hommes (1997) to be used for comparison. Section 3 introduces forward looking behavior with respect to strategy selection. Two different cases will be discussed, one where agents have perfect foresight on expected profits for strategy selection and one where agents make a boundedly rational estimate of expected profits. Section 4 introduces a representative agent who weighs expected profits versus information gathering costs. Section 5 concludes and all proofs are contained in an Appendix.

\section{The original model}

Following Brock and Hommes (1997), we recall some key features of the ARED in the simple economic setting of the cobweb model with selection of forecasting rules based upon past realized profits. The cobweb model describes price fluctuations in a competitive market for a non-storable good that takes one period to be produced. We call $p_{t+1}$ the price of the good at time $t+1$ and $p_{t+1}^{e}$ the expectation at time $t$ of the price at time $t+1$. The demand at time $t+1, D\left(p_{t+1}\right)$, is a linear decreasing function:

$$
D\left(p_{t+1}\right)=A-B p_{t+1}, \quad A>0, B>0 .
$$

The supply at time $t+1, S$, is an increasing function of the producers' forecasts at time $t$ of the price at time $t+1$. More specifically, the supply curve, $S$, is derived from expected profit maximization:

$$
S\left(p_{t+1}^{e}\right)=\operatorname{Argmax}_{x}\left(x p_{t+1}^{e}-c(x)\right),
$$

where $x$ is the amount of goods he decides to produce at time $t$ and $c(x)$ is the production cost function. Because the decision is taken at time $t$, but the price is realized only at time $t+1$, producers have to condition their decision on their expected price $p_{t+1}^{e}$. Taking a quadratic cost function

$$
c(x)=\frac{x^{2}}{2 b}, \quad b>0
$$

gives the linear supply curve: ${ }^{2}$

$$
S\left(p_{t+1}^{e}\right)=b p_{t+1}^{e}
$$

We assume that agents (producers) can choose between two types of predictors, $p_{t+1}^{e, 1}$ and $p_{t+1}^{e, 2}$. Let $n_{t}^{1}$ and $n_{t}^{2}$ be the fractions of agents choosing at the end of time $t$ (or at the beginning of time $t+1$ ), respectively predictor 1 and predictor 2 . The market clearing equation at time $t+1$ is given by:

$$
D\left(p_{t+1}\right)=n_{t}^{1} S\left(p_{t+1}^{e, 1}\right)+n_{t}^{2} S\left(p_{t+1}^{e, 2}\right) .
$$

\footnotetext{
${ }^{2}$ The general case with nonlinear demand and nonlinear supply is investigated in Goeree and Hommes (2000). Because it leads to similar results we restrict our analysis to the linear case.
} 
We now have to specify how agents choose between the two prediction rules. Brock and Hommes (1997) assume that agents are "comparing" past realized profits. At time $t$, the realized profit of strategy $i$ is a function of both the realized price at time $t, p_{t}$, and the predictor $p_{t}^{e, i}, i=1,2$, used at time $t-1$. The price $p_{t}$ determines the price at which the goods are sold, and the predictor $p_{t}^{e, i}$ determines the amount of good produced by agent $i$. As a result, the realized profit at time $t$ under expectations scheme $i$ is given by

$$
\pi_{t}^{i}=\pi\left(p_{t}, p_{t}^{e, i}\right)=p_{t} S\left(p_{t}^{e, i}\right)-c\left(S\left(p_{t}^{e, i}\right)\right)=p_{t} b p_{t}^{e, i}-\frac{\left(b p_{t}^{e, i}\right)^{2}}{2 b}=\frac{b}{2} p_{t}^{e, i}\left(2 p_{t}-p_{t}^{e, i}\right) .
$$

We consider the realistic case where one predictor, say $p^{e, 1}$, is more sophisticated or of higher "quality" than the other predictor $p^{e, 2}$, which is just an easy to use rule of thumb. However, the sophisticated predictor is more "expensive" than the simple predictor because it is more difficult to compute or requires more information gathering. In general, we call $C \geq 0$ the net costs for obtaining the sophisticated predictor.

As mentioned before, the choice of the predictor rule is based on the fitness or performance measure for the two strategies. Brock and Hommes (1997) take last period's net realized profits as the fitness measure; that is, ${ }^{3}$

$$
\begin{aligned}
U_{t}^{1} & =\pi_{t}^{1}-C, \\
U_{t}^{2} & =\pi_{t}^{2}, \\
\Delta U_{t} & =\Delta \pi_{t}-C=\pi_{t}^{1}-\pi_{t}^{2}-C,
\end{aligned}
$$

where $\Delta U_{t}$ is the difference in fitness and $\Delta \pi_{t}$ is the difference in realized profits (ex-cost for the sophisticated rule). The fraction of the agents choosing predictor $i$ at time $t$ is given by a discrete choice (logit) model:

$$
n_{t}^{i}=\frac{e^{\beta U_{t}^{i}}}{Z_{t}}, \quad i=1,2
$$

where $\beta$ is the intensity of choice parameter and $Z_{t}=\sum_{h} e^{\beta U_{t}^{h}}$ is a normalization factor such that $n_{t}^{1}+n_{t}^{2}=1$. The model (7) is derived from a random utility framework; see Brock and Hommes (1997) for more details and Anderson et al. (1993) for many economic applications. The intensity of choice parameter, $\beta$, determines how quickly agents switch to different strategies and it is inversely related to the noise level in the random utility model. In the extreme case $\beta=0$ there is no switching at all and both fractions are 0.5 , whereas in the other extreme case $\beta=\infty$ all agents switch immediately to the best predictor. In an evolutionary framework one can refer to $\beta$ as the selection pressure. As $\beta$ increases the selection pressure increases; that, is more and more agents use the strategy with the higher fitness.

\footnotetext{
${ }^{3}$ More generally, Brock and Hommes (1997) introduce memory in the fitness measure, which is a weighted average of past realized profits. In this paper, we focus on the simplest case with fitness determined by last period realized profit.
} 
If we introduce $m_{t}=n_{t}^{1}-n_{t}^{2}$ we can rewrite the market equilibrium equation as:

$$
A-B p_{t+1}=\frac{b}{2}\left(p_{t+1}^{e, 1}\left(1+m_{t}\right)+p_{t+1}^{e, 2}\left(1-m_{t}\right)\right)
$$

where

$$
m_{t+1}=\tanh \left(\frac{\beta}{2}\left(\Delta \pi_{t+1}-C\right)\right) .
$$

The framework introduced so far is still general in terms of the actual predictors used. Following Brock and Hommes (1997) we consider the choice between costly rational expectations and freely available naive expectations:

$$
\begin{aligned}
& p_{t+1}^{e, 1}=p_{t+1} \\
& p_{t+1}^{e, 2}=p_{t} .
\end{aligned}
$$

The ARED in the case of a rational expectation predictor "versus" a naive predictor is then given by:

$$
\begin{gathered}
A-B p_{t+1}=\frac{b}{2}\left(p_{t+1}\left(1+m_{t}\right)+p_{t}\left(1-m_{t}\right)\right), \\
m_{t+1}=\tanh \left(\frac{\beta}{2}\left[\frac{b}{2}\left(p_{t+1}-p_{t}\right)^{2}-C\right]\right) .
\end{gathered}
$$

The timing in the ARED is important. First, the new market clearing price $p_{t+1}$ is determined using the old fractions $n_{t}^{1}$ and $n_{t}^{2}$ (or difference in fractions $m_{t}$ ). Second, the new realized market price $p_{t+1}$ is used to update and determine the new fractions $n_{t+1}^{1}$ and $n_{t+1}^{2}$ (or difference in fractions $m_{t+1}$ ). Notice that in the market clearing equation $(8) p_{t+1}$ is only implicitly defined, but it can easily be solved explicitly by some map $p_{t+1}=F_{\beta, 1}\left(p_{t}, m_{t}\right)$. The ARED of the cobweb model with rational versus naive expectations and strategy selection based upon realized profits is thus described by a two-dimensional system of nonlinear difference equations $\left(p_{t+1}, m_{t+1}\right)=F_{\beta}\left(p_{t}, m_{t}\right)=\left(F_{\beta, 1}\left(p_{t}, m_{t}\right), F_{\beta, 2}\left(p_{t}, m_{t}\right)\right)$. We discuss the dynamics of $F_{\beta}$ as the intensity of choice, or selection pressure, $\beta$, increases.

A straightforward computation shows that the unique steady state of the system is:

$$
E=\left(p^{*}, m^{*}\right)=\left(\frac{A}{b+B}, \tanh \left(-\frac{\beta C}{2}\right)\right) .
$$

Notice that the steady state value of the difference in fractions, $m^{*}=m^{*}(\beta)$, depends upon $\beta$. In particular, when costs for rational expectations $C>0$, as the intensity of choice $\beta$ increases, the steady-state fraction of rational agents, $n^{1 *}=\left(1+m^{*}\right) / 2$, decreases and $n^{1 *} \rightarrow 0$ as $\beta \rightarrow \infty$. The economic intuition is that at the steady-state $p^{*}$ there is no fitness advantage in buying a rational predictor, because both predictors predict the same, correct, value. As the intensity of choice increases more and more agents choose the naive predictor. The following theorem (theorem 3.1 in Brock and Hommes (1997)) summarizes the stability of the steady state. 
Theorem 1 Assume that the slopes of supply and demand satisfy $b / B>1$ :

(i) When the information costs $C=0$, the steady-state $E=\left(p^{*}, 0\right)$ is always globally stable.

(ii) When the information costs $C>0$, then there exists a critical value $\beta_{1}$ such that for $0 \leq \beta<\beta_{1}$ the steady state is globally stable, while for $\beta>\beta_{1}$ the steady state is an unstable saddle point with eigenvalues 0 and

$$
\lambda(\beta)=-\frac{b\left(1-m^{*}(\beta)\right)}{2 B+b\left(1+m^{*}(\beta)\right)} .
$$

At the critical value $\beta_{1}$ the steady-state value $m^{*}\left(\beta_{1}\right)=-B / b$.

(iii) When the steady state is unstable, there exists a locally unique period 2 orbit $\{(\tilde{p}, \tilde{m}),(-\tilde{p}, \tilde{m})\}$ with $\tilde{m}=-B / b$. There exists a $\beta_{2}>\beta_{1}$ such that the period 2 cycle is stable for $\beta_{1}<\beta<\beta_{2}$.

The assumption $b / B>1$ means that if all agents have naive expectations, the market will be unstable. The case with $b / B<1$ is straightforward because it leads to convergence to the steady state for all values in the parameter space. In the ARED with $b / B>1$, as soon as information costs are positive an increase in the intensity of choice destabilizes the system, and yields an unstable saddle point steady state and the creation of a (stable) 2-cycle through a period doubling bifurcation. Brock and Hommes (1997) show that, as the intensity of choice further increases, the 2-cycle also becomes unstable and a rational route to randomness, that is, a bifurcation route to complicated chaotic price fluctuations, occurs. The mechanism responsible for generating complicated price dynamics is the interplay between a local, close to the steady state, destabilizing force and a global, far from the steady state, stabilizing force. The interplay is fostered by the evolutionary switching mechanism. Technically, it is responsible for homoclinic bifurcations and the presence of strange attractors in the dynamics of prices and fractions, as discussed in detail in Brock and Hommes (1997). Recently, the same mechanism and type of bifurcations have been shown to generate complicated price fluctuations in other frameworks, for example a Cournot duopoly model in Droste et al. (2002) or a financial market where informed and uniformed agents coexist as in de Fontnouvelle (2000) and Diks and Dindo (2006).

The economic intuition of this phenomenon can be explained in the limiting case $\beta=+\infty$. For $\beta=\infty$, in each period, all agents choose the predictor with the highest fitness measure, no matter how small or big this difference is. From (9), for $\beta=\infty$, the difference in fractions $m_{t}$ is determined by:

$$
m_{t}= \begin{cases}+1, & \text { if } \quad \frac{b}{2}\left(p_{t}-p_{t-1}\right)^{2}>C \\ -1, & \text { if } \quad \frac{b}{2}\left(p_{t}-p_{t-1}\right)^{2} \leq C\end{cases}
$$

so that $p_{t+1}$ is given by:

$$
p_{t+1}=f_{\infty}\left(p_{t}, p_{t-1}\right)=\left\{\begin{array}{ll}
p^{*}, & \text { if } \quad \frac{b}{2}\left(p_{t}-p_{t-1}\right)^{2}>C \\
\frac{A}{B}-\frac{b}{B} p_{t}, & \text { if } \quad \frac{b}{2}\left(p_{t}-p_{t-1}\right)^{2} \leq C
\end{array} .\right.
$$


The following theorem (theorem 3.2 in Brock and Hommes (1997)) characterizes the price dynamics in this case.

Theorem 2 For $\beta=\infty$, even when the market is locally unstable (i.e. $b / B>1$ ) and when information costs $C>0$, the system always converges to the saddle point equilibrium steady state $E=\left(p^{*},-1\right)$.

The reasoning behind the proof is quite instructive and provides a simple economic intuition. For $\beta=\infty$ in each period either all agents are rational $(m=+1)$ or all agents are naive $(m=-1)$. Assume, for example, that all agents are naive and let the price be close to the steady state. For $m=-1$ the price will diverge from $p^{*}$ due to the local instability. As long as $m=-1$, price fluctuations become bigger (in absolute value) and errors made by the naive forecast will rapidly increase, until the point where the difference between realized profits of rational and naive expectations exceeds the costs for rational expectations. At this point, because $\beta=\infty$, all agents buy the rational predictor, so that $m$ becomes +1 , and in the next period the price jumps immediately onto the steady-state price $p^{*}$, and remains there forever.

For future comparison with the model of Section 3, it is instructive to compute the difference between the maximum and the minimum observable price when $\beta=\infty$. We define this maximum difference as $\Delta_{\infty}^{f}$, where the superscript $f$ and the subscript $\infty$ refer to the map $f_{\infty}$ defined in (10).

Lemma 1 When $\beta=\infty$, the difference between the maximum and the minimum observable price is $\Delta_{\infty}^{f}=2 \frac{b^{2}}{B(B+b)} \sqrt{2 C / b}$.

\section{Strategy switching with forward looking agents}

In the original Brock and Hommes model, agents are backward looking in their selection of prediction strategies because their choice is based upon past realized profits, but they are forward looking in their production decision because, given their price forecast, they maximize expected profits. In this section we discuss the model with forward looking agents, with both strategy selection and production decisions based upon expected profit. In the forward looking case, at time $t$ the fitness measure difference used for strategy selection between the rational and naive predictors becomes:

$$
\Delta U_{t+1}^{e}=\Delta \pi_{t+1}^{e}-C=\pi_{t+1}^{e, 1}-\pi_{t+1}^{e, 2}-C
$$

where, as before, $C$ represents the costs for the rational expectations predictor.

We will discuss two different versions of the model with forward looking agents, depending on the way agents compute their expected profit. In Subsection 3.1 all agents (i.e. both agents using the rational and the naive forecast) have perfect foresight on expected profits conditional on their production decision. We will refer to this case as perfectly forward looking agents in strategy selection. In Subsection 3.2 we discuss a different version of the model where agents using the rational price forecast still have perfect foresight on expected profits, whereas the agents using the naive price forecast use their most recent observations 
to make a simple estimate of their expected profit. We will refer to this case as boundedly rational forward looking agents.

\subsection{Perfectly forward looking agents}

Consider forward looking agents and a discrete choice random utility model, with expected profit as the fitness measure in the following way. As before, given the expected price $p_{t+1}^{e}$, the optimal supply $S\left(p_{t+1}^{e}\right)=b p_{t+1}^{e}$ is derived from expected profit maximization with quadratic production cost function. There are two price forecast strategies available, the rational forecast at cost $C$ and the free naive forecast. As before, market equilibrium at time $t+1$ is given by:

$$
A-B p_{t+1}=n_{t}^{1} b p_{t+1}+n_{t}^{2} b p_{t} .
$$

The fraction of agents who adopt strategy $i, n_{t}^{i}$, is determined through a discrete choice model as in (7), but this time the fitness of each predictor is measured in terms of the expectations at time $t$ about profits at time $t+1$ :

$$
n_{t}^{i}=\frac{e^{\beta U_{t+1}^{e, i}}}{Z_{t}},
$$

where $Z_{t}=\sum_{h} e^{\beta U_{t+1}^{e, h}}$ is the usual normalization factor. The rational predictor is evaluated according to:

$$
U_{t+1}^{e, 1}=\pi_{t+1}^{e, 1}-C,
$$

whereas the naive predictor is evaluated according to:

$$
U_{t+1}^{e, 2}=\pi_{t+1}^{e, 2} \text {. }
$$

In both cases:

$$
\pi_{t+1}^{e, i}=\pi\left(p_{t+1}, p_{t+1}^{e, i}\right)=p_{t+1} S\left(p_{t+1}^{e, i}\right)-c\left(S\left(p_{t+1}^{e, i}\right)\right) .
$$

Notice that the expected profit, $\pi_{t+1}^{e, i} i=1,2$, depends both on the expected price $p_{t+1}^{e, i}$, which determines the production decision at time $t$, and on the price that clears the market at time $t+1, p_{t+1}$. We assume that the economy works "as if" agents have perfect foresight on this second price. This implies that whatever their production decision is, agents have perfect foresight on expected profits. Stated differently, given the production decisions, fractions of the strategies are determined "as if" agents compute expected profits without errors.

At this point it is useful to discuss a potential inconsistency problem in the model. If agents have perfect foresight on the expected profit, then, at time $t$, wouldn't they also "know" the price forecast $p_{t+1}$ ? If they could extract the perfect foresight price forecast from perfect foresight on expected profits, there would be no incentive left to pay the information cost $C$ for the perfect price forecast. That is, why buy the cow when you can get the milk for nothing? 
To avoid this behavioral inconsistency, we assume that there is an expert manager who has "structural knowledge" about the economy enabling him to have structural rational expectations. Each agent can choose either to run the business by himself or hire an expert manager with "structural knowledge" of the economy and rational expectations. The manager promises the agent a sure net profit, after subtracting an (unknown) cost $C$ for his service. Agents take the decision to hire or not to hire the rational expert manager by evaluating the fitness measure difference (11), which is computed and announced by the expert manager and available as public information. The fraction of agents that chooses to hire the rational expert manager is determined by the difference in fitness announced by the manager, according to the random utility framework. It is important that agents do not know the division between profits and costs $C$ in the net revenue they receive from their manager, because if they did they could derive the perfect foresight forecast from public information. Notice also that the manager is "credible" in the sense that, given the production decision of the agents, the manager delivers the net profits that he announces.

We are aware of the limitations of these assumptions and we do not claim this to be a realistic description of market behavior. Rather, we view this model as an interesting theoretical benchmark with strategy switching determined by rational forward looking behavior on expected profits. In this theoretical benchmark, forward looking strategy switching is not affected by any mistakes of the agents in evaluating their expected profits. In Subsection 3.2 we will consider the probably more realistic case where some forward looking agents make mistakes in evaluating their expected payoff.

Under the assumption of perfect foresight on expected profits for both types, the expected profits for rational respectively naive agents are given by

$$
\begin{gathered}
\pi_{t+1}^{e, 1}=\pi\left(p_{t+1}, p_{t+1}^{e, 1}\right)=p_{t+1} S\left(p_{t+1}\right)-c\left(S\left(p_{t+1}\right)\right)=\frac{b}{2} p_{t+1}^{2}, \\
\pi_{t+1}^{e, 2}=\pi\left(p_{t+1}, p_{t+1}^{e, 2}\right)=p_{t+1} S\left(p_{t}\right)-c\left(S\left(p_{t}\right)\right)=\frac{b}{2} p_{t}\left(2 p_{t+1}-p_{t}\right),
\end{gathered}
$$

where we used $p_{t+1}^{e, 1}=p_{t+1}$ and $p_{t+1}^{e, 2}=p_{t}$. Notice that the fitness difference becomes (11):

$$
\Delta U_{t+1}^{e}=\frac{b}{2}\left(p_{t+1}-p_{t}\right)^{2}-C .
$$

The fractions of the two types are determined through a discrete choice model as before, with fitness measure difference (19). Working again with the difference in fractions $m_{t}=$ $n_{t}^{1}-n_{t}^{2}$, the system is given by:

$$
\begin{gathered}
A-B p_{t+1}=\frac{b}{2}\left(p_{t+1}\left(1+m_{t}\right)+p_{t}\left(1-m_{t}\right)\right), \\
m_{t}=\tanh \left(\frac{\beta}{2}\left[\frac{b}{2}\left(p_{t+1}-p_{t}\right)^{2}-C\right]\right) .
\end{gathered}
$$




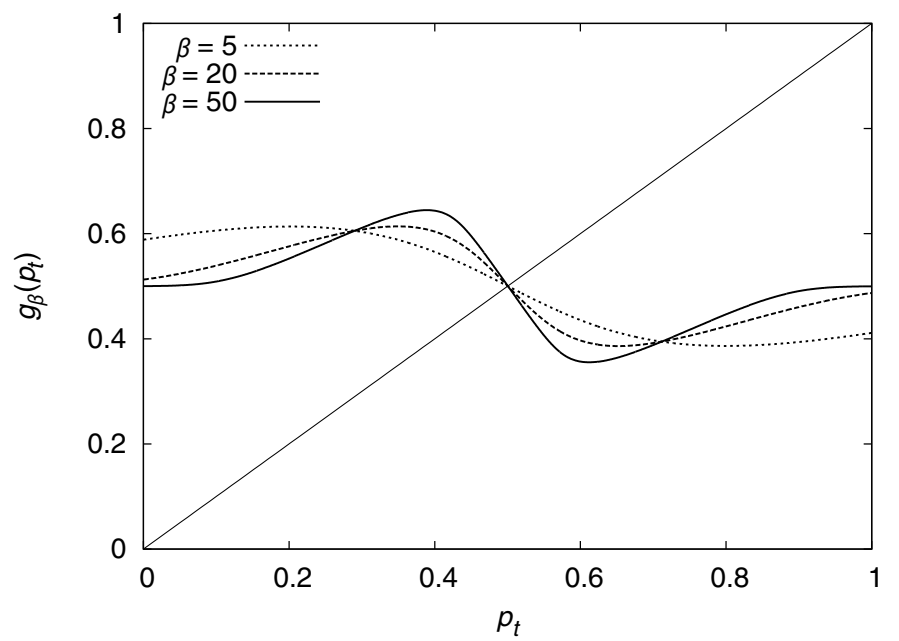

Figure 1 Perfectly forward looking agents. The graph of $p_{t+1}=g_{\beta}\left(p_{t}\right)$ for three different values of $\beta$. The three points where all graphs intersect correspond to the steady-state $p^{*}$ (in the middle) and points where $m\left(p_{t}, p_{t+1}\right)=0$; that is, where the profit difference of the two strategies is equal to the net cost, $C$. Other parameters are: $A=1.5, B=1, b=2, C=0.1$.

We define a managerial perfect foresight equilibrium to be a time path of prices $p_{t}$, produced quantities $x_{t}^{i}, i=1,2$, fractions of agents choosing strategy $i, n_{t}^{i}, i=1,2$, and point expectation of profits, $\pi_{t+1}^{e, i}, i=1,2$, that at each date $t$ satisfies equations (12-18) with supply equals demand and fulfilled point expectations. Co-evolution of prices and (difference in) fractions is described by (20) and (21). We use the extra adjective, managerial, because our concept of perfect foresight equilibrium requires both agents having perfect foresight (i.e. fulfilled point expectations) on the amount of net revenue (which is the net profit received by the agent after deducting the manager's operating cost, $C$ ), as well as the manager generating profits consistent with perfect foresight on the date $t+1$ price $p_{t+1}$.

It is useful to compare the model with perfectly forward looking agents (20) and (21) to the original model with backward looking agents (8) and (9). In the forward looking case the difference in fractions $m_{t}$ is simply a one period ahead version of the backward looking case. In fact, in the forward looking case the difference in fractions of the two strategies, $m_{t}$, depends upon $p_{t}$ and $p_{t+1}$, implying that the market clearing equation (20) is only implicitly defined. The following result states that (20) and (21) translate into a well defined, explicit one-dimensional map $p_{t+1}=g_{\beta}\left(p_{t}\right)$ (see Figure 1).

Theorem 3 Given a linear demand and a linear supply curve, for any value of the information $\operatorname{cost} C \geq 0$, the intensity of choice $\beta \geq 0$ and the initial price $p_{t}$, (20) and (21) determine a well defined map $p_{t+1}=g_{\beta}\left(p_{t}\right)$. That is, there exists a unique non-negative price $p_{t+1}$ such that, at time $t+1$, either the market is in equilibrium or the excess supply is positive at $p_{t+1}=0$. Furthermore, the map $g_{\beta}$ is continuous. 
A straightforward calculation shows that, as before, $p^{*}=A /(b+B)$ is always the unique fixed point of the map $g_{\beta}$. The corresponding fraction difference $m$ is again given by $m^{*}(\beta)=\tanh (-\beta C / 2)$. The following theorem characterizes the dynamics:

Theorem 4 Assume that the slopes of supply and demand satisfy $b / B>1$.

(i) When the information costs $C=0$, the steady state $E=p^{*}$ is always globally stable.

(ii) When the information costs $C>0$, then there exists a critical value $\beta_{1}$ such that for $0 \leq \beta<\beta_{1}$ the equilibrium is globally stable, whereas for $\beta>\beta_{1}$ the equilibrium is unstable with eigenvalue

$$
\lambda(\beta)=-\frac{b\left(1-m^{*}(\beta)\right)}{2 B+b\left(1+m^{*}(\beta)\right)} .
$$

At the critical value $\beta_{1}$ the steady-state value $m^{*}\left(\beta_{1}\right)=-B / b$.

(iii) When the steady state is unstable, there exists a unique period 2 orbit $\left\{p_{1}, p_{2}\right\}$. The period 2 orbit is globally stable for any value of $\beta>\beta_{1}$.

Compare the local stability of the system with forward looking agents as specified in Theorem 4 with the local stability of the system with backward looking agents as specified in Theorem 1. Points $(i)$ and ( $i$ i ) of both Theorems imply that for small values of the switching parameter $\beta$ the systems behave similarly. When the switching parameter is smaller than the primary bifurcation value $\beta_{1}$ the steady state is stable, whereas for $\beta>\beta_{1}$ a (stable) period 2-cycle is created. Notice that the primary bifurcation value $\beta_{1}$ is the same for both systems. For larger values of $\beta$, according to (iii), the backward looking and forward looking cases are different. In the backward looking case the 2-cycle is stable only when $\beta_{1}<\beta<\beta_{2}$, whereas in the perfectly forward looking case the 2 -cycle is stable for all values of the switching parameter $\beta>\beta_{1}$. The rational route to randomness (i.e. the bifurcation route to chaos) has disappeared because of the perfectly forward looking strategy switching behavior of the agents. Technically, no homoclinic bifurcation is observed and the interplay between local instability and global stability is different.

The reason that complicated dynamics does not arise is that perfectly forward looking agents do not make mistakes in the estimation of their expected profits. In contrast, backward looking agents use past realized profits to evaluate which predictor to use, and, therefore, might make big mistakes. In particular, these mistakes might lead to big losses for the agents who choose a naive predictor, especially when $\beta$ is high. In fact, in the backward looking case, when the cheap naive predictor has performed well in the last period it attracts many agents. This triggers local instability with prices deviating more and more from the steady state and bigger and bigger errors in profit estimation. When these mistakes become sufficiently large and $\beta$ is high, many agents switch back to the rational predictor, therefore pushing prices back very close to the steady state. However, close to the steady state the cheap naive predictor works fine and is less costly than the rational predictor, so that the story repeats. When agents are perfectly forward looking concerning expected profits, big mistakes by the naive predictor are anticipated by the forward looking behavior and, therefore, prices will not deviate very far from their steady state nor will they be pushed back very close to the steady state. 


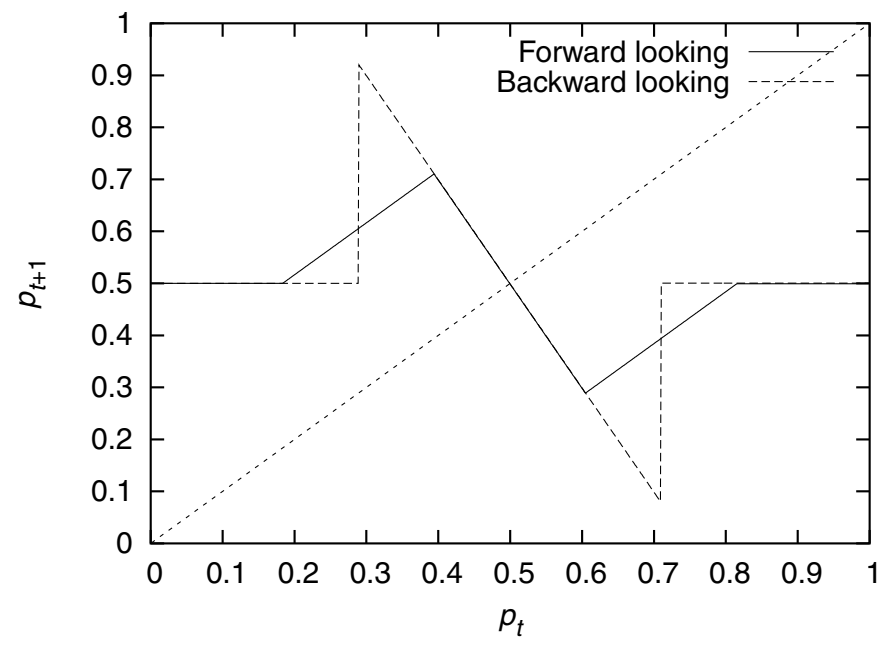

Figure $2 \beta=\infty$. Comparison of the map $p_{t+1}=g_{\infty}\left(p_{t}\right)$ in (22) for perfectly forward looking agents and the corresponding one-dimensional map in the case of backward looking agents. The latter has been obtained from the projection of the two-dimensional map $p_{t+1}=f_{\infty}\left(p_{t}, p_{t-1}\right)$ in (10) with $p_{t-1}$ given by the inverse-image of $\left(p_{t},-1\right)$ through the map $F_{\beta}, 1$ (see also the proof of Lemma 1 in Appendix 6.1). Other parameters: $A=1.5, B=1, b=2 C=0.1$.

An analysis of the case of an infinite switching parameter enables us to make this point precise. Remember that in the case of backward looking agents for $\beta=\infty$ the price always converges to the (locally unstable) steady state value, as stated in Theorem 2. As we shall prove in the next theorem in the case of forward looking agents, in the limit case $\beta=\infty$ prices always converge to a period 2 orbit. For $\beta=\infty$ the map $g_{\beta}$ defined by (20) and (21) becomes (see Figure 2):

$$
p_{t+1}=g_{\infty}\left(p_{t}\right)= \begin{cases}p^{*}, & p_{t} \in\left[0, p^{*}-\tilde{p}\right] \cup\left[p^{*}+\tilde{p}, \infty\right) \\ p_{t}+\sqrt{2 C / b} & p_{t} \in\left(p^{*}-\tilde{p}, p^{*}-\delta \tilde{p}\right) \\ \frac{A}{B}-\frac{b}{B} p_{t}, & p_{t} \in\left[p^{*}-\delta \tilde{p}, p^{*}+\delta \tilde{p}\right] \\ p_{t}-\sqrt{2 C / b} & p_{t} \in\left(p^{*}+\delta \tilde{p}, p^{*}+\tilde{p}\right)\end{cases}
$$

where $\tilde{p}=\sqrt{2 C / b}$ and $\delta=B /(B+b)<1$.

Theorem 5 For $\beta=\infty$, when the market is locally unstable (i.e. $b / B>1$ ) and when information costs $C>0$ the system always converges to a period 2 orbit. A continuum of period 2 orbits exists. Furthermore, the difference between the maximum and the minimum observable price is:

$$
\Delta_{\infty}^{g}=2 \frac{b}{B+b} \sqrt{2 C / b}
$$

that is, $\Delta_{\infty}^{g}=\left(\frac{B}{b}\right) \Delta_{\infty}^{f}<\Delta_{\infty}^{f}$. 
The last part of Theorem 5 points to another difference with the original model concerning the size of fluctuations. Figure 2 illustrates this difference between the models with backward looking and forward looking strategy selection. The backward looking map $f_{\infty}$ has a higher maximum and a lower minimum; that is, $\Delta_{\infty}^{g}<\Delta_{\infty}^{f}$. The comparison of the two maps clarifies that the globally stabilizing forces are different. In the backward looking case agents make larger errors and it takes one extra time period before agents are ready to switch to the costly rational strategy. Moreover, if the errors have grown too large, all agents share the same experience and all switch to the costly rational strategy at the same time. In the forward looking case, agents anticipate large mistakes and start switching to the rational strategy before the errors grow too large. Moreover, forward looking behavior prevents all agents switching at the same point in time, but instead ensures a smooth and gradual switching to the costly rational strategy, leading to smooth dynamics and an interval of 2-cycles at some not too far away distance from the steady state with rational and naive agents co-existing (see the parts of the graph of $g_{\infty}$ parallel to the diagonal). In this model, forward looking behavior based on expected profits instead of realized profits leads to smoother transitions and dampened fluctuations in the short run but also prevents the system from returning (close) to the steady state price in the long run.

The presence of a regular 2-cycle for a large interval of values of the switching parameter $\beta$ (e.g. Figure 3) raises the question of whether boundedly rational agents are able to detect the regular structure from time series observations and exploit it? Stated differently, is the 2-cycle equilibrium "evolutionary stable"; that is, will the cycle persist when other boundedly rational agents "invade" the system? We will not address this problem in detail here, but investigate the question using some simple simulations, illustrated in Figure 4. The answer depends on whether or not additional boundedly rational forecasting rules have costs associated with them, (e.g. higher costs when the rule uses more memory). In what follow, all agents, including those using the new price forecasting rules, have perfect foresight on expected profits as before. First consider the case where rational expectations at cost $C$ versus free naive expectations leads to a stable 2-cycle. Suppose a new forecasting rule, $p_{t+1}^{e}=p_{t-1}$ (call it a period-2 rule), at costs $C^{\prime}, 0<C^{\prime}<C$, enters the system. Along the 2-cycle this period-2 rule has in fact perfect foresight, and because it is cheaper than the rational perfect foresight rule, many agents will start using the period- 2 rule. Figure 4 shows that the system with rational versus period-2 versus naive locks into another stable 2-cycle, with smaller amplitude because of the lower costs for the period-2 rule. The costly rational expectations rule is (almost) driven out of the market and replaced by the cheaper (but still costly) period-2 rule, but the long-run equilibrium outcome remains a stable 2-cycle (with smaller amplitude).

Next consider the case where costly rational versus free naive expectations leads to a stable 2-cycle, and a new period-2 rule at zero costs invades the system. Figure 4 shows that the system with costly rational versus free naive versus free period-2 converges to a stable 3 -cycle of smaller amplitude. The intuition is that along the original 2-cycle, agents switch to the period-2 rule because its forecast is the same as the rational rule but at no costs. As a consequence, the amplitude of price fluctuations decreases, which leads to smaller forecasting errors of the naive rule and, therefore, an increase of the fraction of agents using the naive rule. With the naive and the period- 2 rule both having positive fractions the 

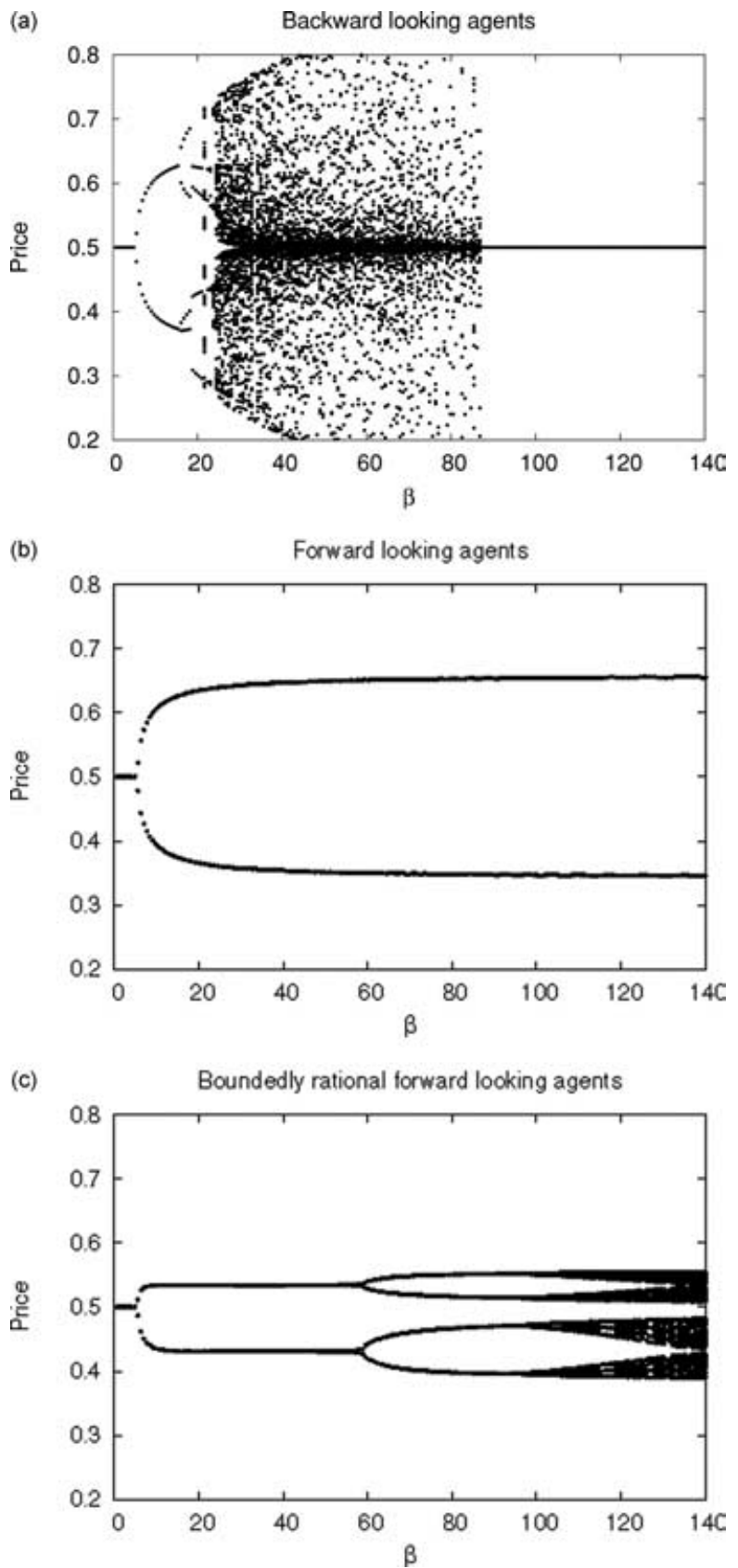

Figure 3 Bifurcation diagrams with respect to $\beta$. The case of (a) backward looking agents (Section 2), (b) perfectly forward looking agents (Section 3.1) and (c) the case of boundedly rational forward looking agents (Section 3.2). The other parameters are $A=1.5, B=1.0, b=2.0$ (so that $\left.p^{*}=0.5\right)$ and $C=0.1$. 
(a)

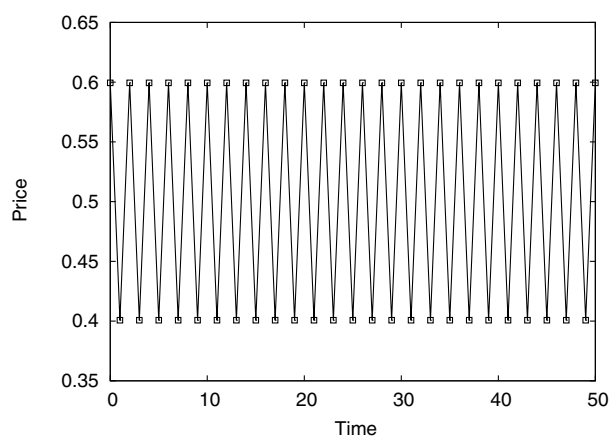

(c)

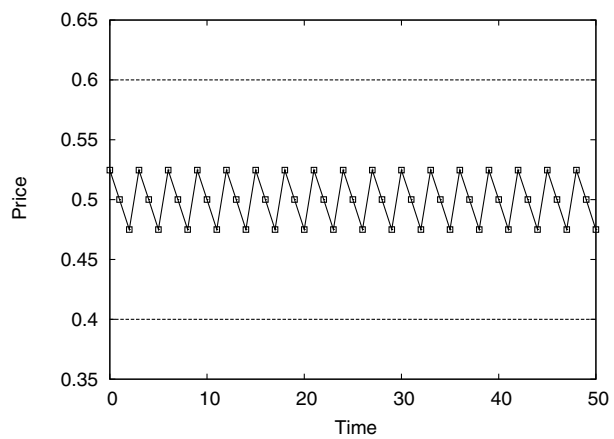

(e)

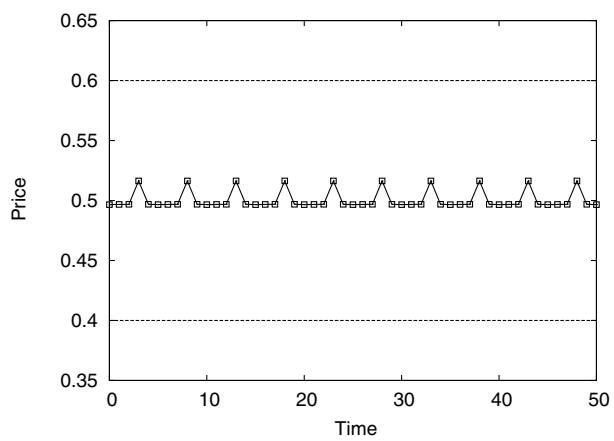

(b)

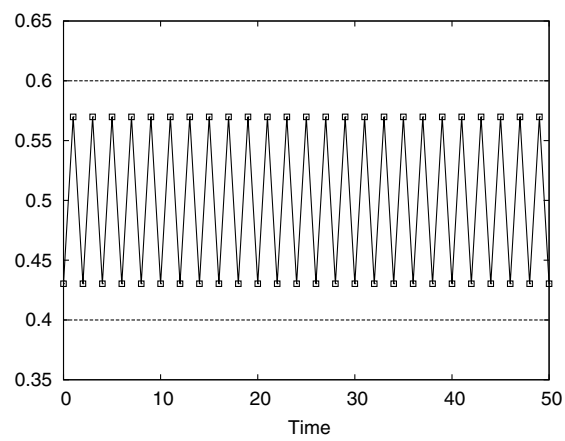

(d)

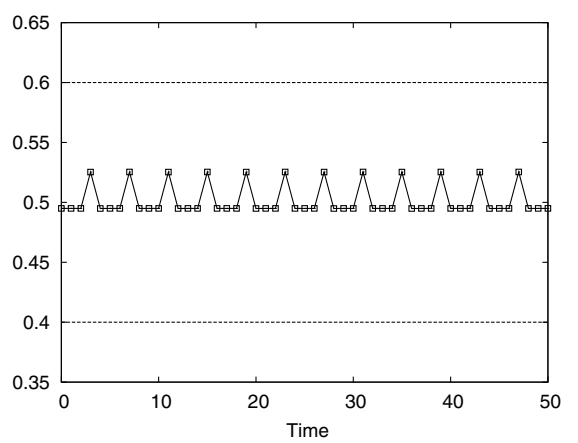

(f)

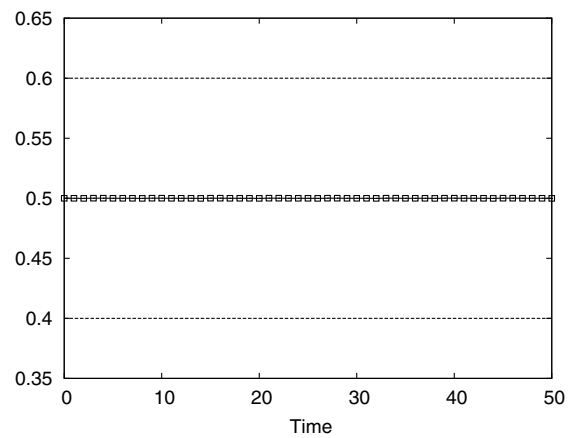

Figure 4 Perfectly forward looking agents. This figure illustrates the persistence of the stable 2-cycle when free naive and costly rational predictors are available (a) with respect to "invasion" of other predictors (b-f). If a period-2 predictor emerges at a cost $0<C^{\prime}<C$, the stable 2 -cycle persists but with smaller amplitude (b). If a period-2 predictor invades at no cost, the interaction of naive, rational and period-2 predictors leads to a 3-cycle (c). In (a)-(c), at every step (from left to right and from top to bottom) a higher period predictor is introduced. This leads to the creation of a stable 4-cycle, a stable 5-cycle and, finally, to the stabilization of the steady state. In all cases, parameters are

$$
A=3, B=1, b=5, \beta=300, C=0.1 \text {. }
$$


system locks into a stable small amplitude 3-cycle. We can continue the story and introduce an additional type using a period-3 rule (i.e. $p_{t+1}^{e}=p_{t-2}$ ) entering the market. This system with four different rules locks into a stable 4-cycle with even smaller amplitude, as shown in Figure 4. Similarly, adding a new fifth type, a period-4 rule, the system locks into a stable 5-cycle with very small amplitude. Finally, adding a period-5 rule, the system stabilizes and locks into the stable steady state price. The intuition is that with more and more period-k forecasting rules at no cost, many rules gain positive weight and the system behaves as if agents use (a weighed average of) past prices as their forecast, therefore stabilizing price fluctuations.

The key point of this simulation exercise is that when new boundedly rational forecasting rules "invade" the system and more memory comes at higher costs, the stable 2-cycle persists but has smaller amplitude. In contrast, when there are no costs associated to boundedly rational rules with more memory, price fluctuations stabilize. This situation is similar to the simulation experiments of the El-Farol bar problem in Arthur (1994), where "cycles are quickly arbitraged away" by cycle-detector predictors. Another example, where autocorrelation structure is arbitraged away by simple rules, is presented in Dindo and Tuinstra (2006). They argue that this might be a characteristic feature of systems with negative feedback from expectation to realization, as in the cobweb model.

\subsection{Boundedly rational forward looking agents}

In the previous subsection all agents were perfectly forward looking concerning expected profits. In this subsection we back off from perfect rationality and assume that to choose their strategy some agents only make a boundedly rational estimate of expected profits. As in the previous subsection, we assume that each agent is faced with the choice of either hiring an expert manager to run the business or running the business themself. In the first case, the story unfolds as in the previous subsection: by hiring an expert manager, the owner is promised at time $t$ to receive at time $t+1$ a sure profit $U_{t+1}^{e, 1}$ as computed in (14) and (17). The manager promising this last payoff uses rational expectation and keeps an (unknown) amount $C$ of the profit of the firm for himself. As a result, $U_{t+1}^{e, 1}=\pi_{t+1}^{e, 1}-C$. Because the agent (the owner of the firm) does not know $C$, he is not able to extract the rational expectation price forecast at the time he compares the two expected net profits. The computation of the fitness in the second case, when the owner decides to run the business by himself, is done in a different way than in the previous subsection. Equation (15) still holds but, in this case, we assume that the naive agent makes an estimate of the expected profit. We refer to this case as boundedly rational forward looking agents. We focus here on a simple case where the naive agents use their naive forecast both in the production decision and in the forecast of the expected profit. Instead of (18) he uses:

$$
\pi_{t+1}^{e, 2}=\pi\left(p_{t+1}^{e, 2}, p_{t+1}^{e, 2}\right)=\pi\left(p_{t}, p_{t}\right)=\frac{b}{2}\left(p_{t}\right)^{2} .
$$

In Subsection 3.1 agents switch between prediction strategies only because one of the two is granted a higher profit. In the present case, agents switch between the two predictors 
not only because naive agents make price forecasting errors, but also because they make a wrong estimate of the expected payoff they will get by using the naive predictor. As we shall see, this additional error complicates the qualitative dynamics, but does not change the amplitude of the price fluctuation significantly.

Summing up, at time $t$, the expected fitness difference of the two predictors is:

$$
\Delta U_{t+1}^{e}=\frac{b}{2}\left(p_{t+1}^{2}-p_{t}^{2}\right)-C .
$$

The fraction of agents using the predictor $i=1,2$ at time $t$ is as in (13). Using $m_{t}=n_{t}^{1}-n_{t}^{2}$, market equilibrium and the evolution of the fractions' difference are

$$
\begin{gathered}
A-B p_{t+1}=\frac{b}{2}\left[p_{t+1}\left(1+m_{t}\right)+p_{t}\left(1-m_{t}\right)\right], \\
m_{t}=\tanh \left(\frac{\beta}{2}\left[\frac{b}{2}\left(p_{t+1}^{2}-p_{t}^{2}\right)-C\right]\right) .
\end{gathered}
$$

As in the case of perfectly forward looking agents in (21), the difference in fractions $m_{t}$ in (26) depends upon $p_{t}$ and $p_{t+1}$, so that the market clearing equation (25) is again implicitly defined. The following result states that (25) and (26) determine a well defined, explicit one-dimensional map $p_{t+1}=h_{\beta}\left(p_{t}\right)$ :

Theorem 6 Given linear demand and linear supply, for any value of the information cost $C \geq 0$, the intensity of choice $\beta \geq 0$ and the price $p_{t}$, the system ((25) and (26)) implies a well defined map $p_{t+1}=h_{\beta}\left(p_{t}\right)$. That is, there exists a unique minimum non-negative price $p_{t+1}$ such that, at time $t+1$, either the market is in equilibrium or the excess supply is positive at $p_{t+1}=0$. Sufficient conditions for continuity of the map $h_{\beta}$ are $\beta<4 /\left(A p^{*}\right)$ or, when $\beta>4 /\left(A p^{*}\right)$,

$$
1+\tanh \left(-\frac{\beta}{2} C\right)<2 \frac{B}{b} \frac{4 /\left(A p^{*}\right)}{\beta-4 /\left(A p^{*}\right)} .
$$

Notice that (27) is always satisfied in the limit $\beta \rightarrow \infty$. Some graphs of the map $h_{\beta}$ and the associated dynamics are given in Figure 5. The fixed point of the system is the same as before: $E=\left(p^{*}, m^{*}(\beta)\right)=(A /(b+B), \tanh (-\beta C / 2))$. The following theorem is the analogue of Theorems 1 and 4 in the case of forward looking boundedly rational agents:

Theorem 7 Assume that the slopes of supply and demand satisfy $b / B>1$.

(i) When the information costs $C>0$, then there exists a critical value $\beta_{1}$ such that for $0 \leq \beta<\beta_{1}$ the equilibrium is globally stable, whereas for $\beta>\beta_{1}$ the equilibrium is unstable with eigenvalue

$$
\lambda(\beta)=-\frac{b\left(1-m^{*}(\beta)\right)}{2 B+b\left(1+m^{*}(\beta)\right)} .
$$

At the critical value $\beta_{1}$ the steady state value $m^{*}\left(\beta_{1}\right)=-B / b$.

(ii) When the steady state is unstable, there exists a locally unique period 2 orbit $\left\{p_{1}, p_{2}\right\}$. 

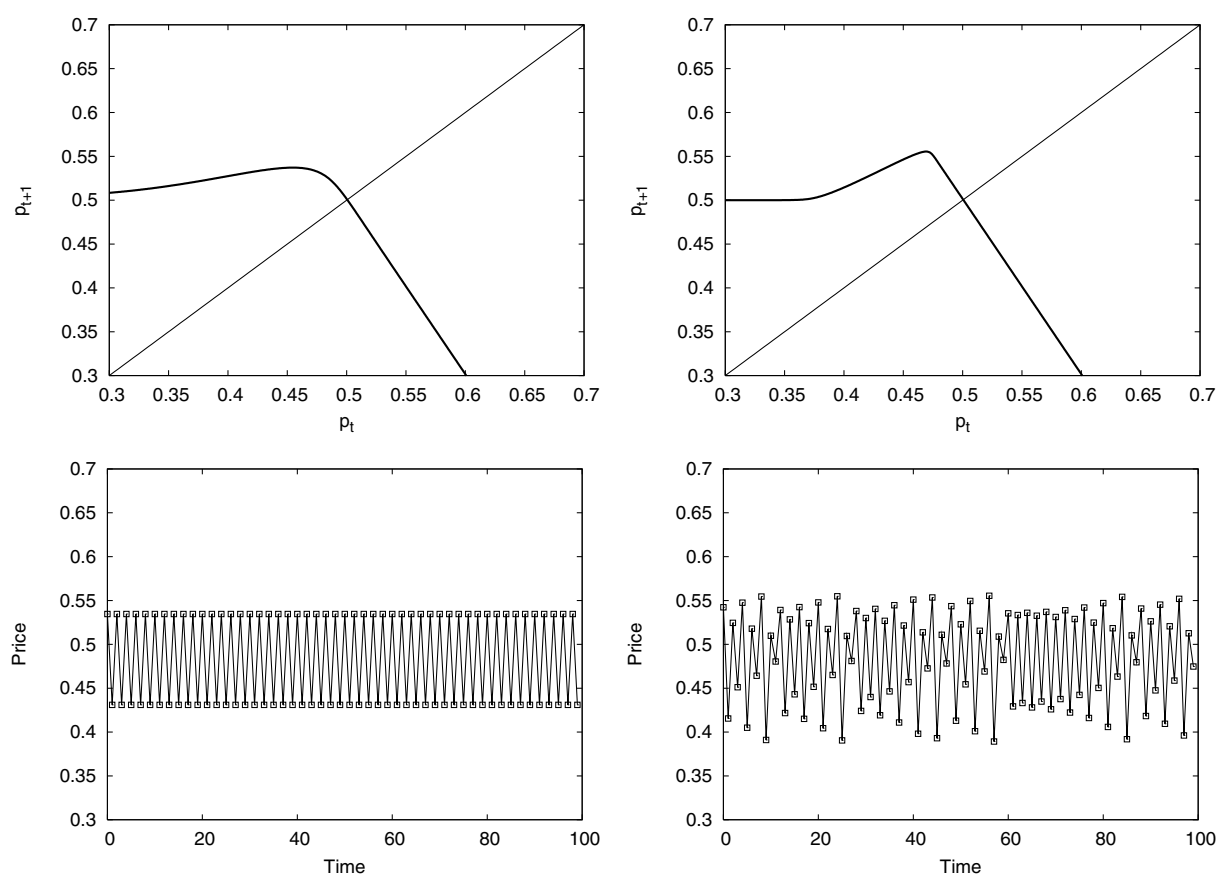

Figure 5 Boundedly rational forward looking agents. On the left: map and price dynamics for $A=1.5, B=1.0, b=2.0, C=0.1$ and $\beta=20$. The price dynamics converges to a 2-cycle. On the right: map and price dynamics for $A=1.5, B=1.0, b=2.0, C=0.1$ and $\beta=140$. The price dynamics is chaotic.

Notice the similarity between Theorems 1, 4 and 7. In all cases, the primary bifurcation leading to local instability is the same. However, after local instability sets in, the global dynamics becomes quite different. Figures 3 and 6 compare the dynamics of all three cases, the original backward looking case, the perfectly forward looking case and the boundedly rational forward looking case. Both forward looking cases clearly have price fluctuations with smaller amplitude, as explained already in Subsection 3.1. In contrast to the perfectly forward looking case, with boundedly rational forward looking agents simulations show that a secondary bifurcation and a rational route to randomness (i.e. a bifurcation route to chaos) occur, but for higher values of $\beta$ than in the original Brock and Hommes model and with chaotic fluctuations with smaller amplitude. The investigation of the case $\beta=\infty$ helps us to understand the origins of this difference, the intuition behind it and its economic consequences.

For $\beta=\infty$, the difference in fractions (26) becomes:

$$
m_{t+1}=\left\{\begin{array}{cc}
+1, & \text { if } \quad \frac{b}{2}\left(p_{t+1}^{2}-p_{t}^{2}\right)>C \\
-1, & \text { if } \quad \frac{b}{2}\left(p_{t+1}^{2}-p_{t}^{2}\right)<C,
\end{array}\right.
$$


(a)

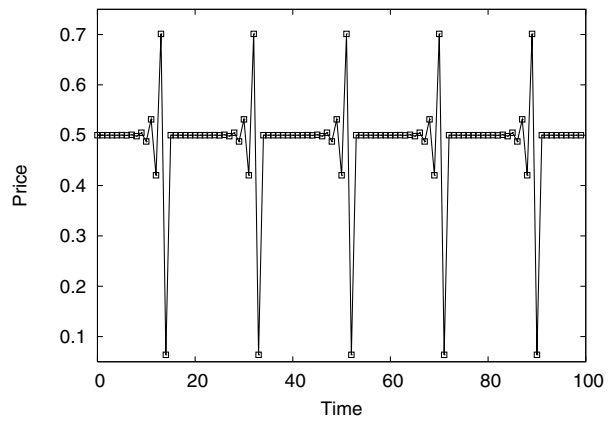

(b)

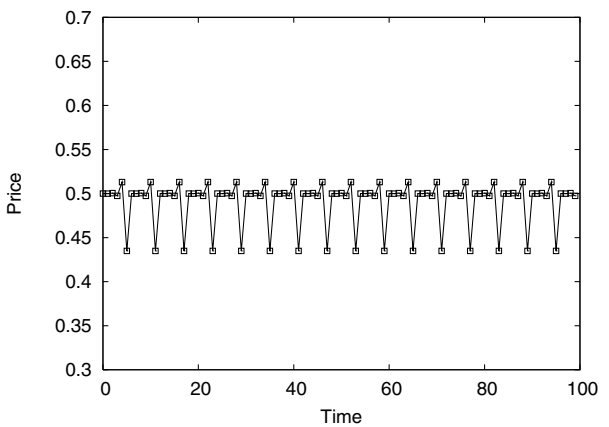

Figure 6 Comparison of the cases with backward looking agents (a) and boundedly rational forward looking agents (b). In both cases, there exist homoclinic points converging both forward and backward in time to the steady state. As a consequences in both cases at irregular time intervals price fluctuations approach the (locally unstable) steady state. The amplitude of price oscillations is much smaller in the forward looking case. In both cases the parameters are: $A=1.5, B=0.85, b=2.15$ (so that $p^{*}=0.5$ ), $\beta=200$ and $C=0.1$.

and, using (25), the price at time $t+1$ becomes

$$
p_{t+1}= \begin{cases}p^{*}, & \text { if } p_{t+1}>\sqrt{p_{t}^{2}+2 C / b} \\ \frac{A}{B}-\frac{b}{B} p_{t}, & \text { if } p_{t+1}<\sqrt{p_{t}^{2}+2 C / b} \\ \sqrt{p_{t}^{2}+2 C / b} & \text { otherwise. }\end{cases}
$$

If we define $\bar{p}$ such that $\sqrt{\bar{p}^{2}+2 C / b}=p^{*}$ and $\hat{p}$ such that $A / B-(b / B) \hat{p}=$ $\sqrt{\hat{p}^{2}+2 C / b}$, the map $h_{\infty}$ becomes: ${ }^{4}$

$$
p_{t+1}=h_{\infty}\left(p_{t}\right)= \begin{cases}p^{*}, & p_{t}<\bar{p} \\ \sqrt{p_{t}^{2}+2 C / b}, & p \in[\bar{p}, \hat{p} .] \\ \frac{A}{B}-\frac{b}{B} p_{t}, & p_{t}>\hat{p} .\end{cases}
$$

Two graphs of the map $h_{\infty}$ are given in Figure 10 in the Appendix illustrating the proofs. The following theorem states that, if the market is sufficiently unstable, in the case with boundedly rational agents complicated dynamical behavior arises.

Theorem 8 For $\beta=+\infty$, when the market is locally unstable (i.e. $b / B>1$ ) and when information costs $C>0$, there exists a value $M>1$ (depending on $p^{*}, C$ and $b$ ) such that:

(i) When $b / B \in(1, M]$, for an interval of initial conditions, the prices dynamics is bounded away from $p^{*}$.

(ii) When $b / B>M$, there exist infinitely many homoclinic points $p$, that is, points such that $\lim _{n \rightarrow+\infty} h_{\infty}^{n}(p)=p^{*}$ and $\lim _{n \rightarrow-\infty} h_{\infty}^{n}(p)=p^{*}$.

\footnotetext{
${ }^{4}$ Notice that $\bar{p}$ is only defined if $C$ is not too large. If $\bar{p}$ is well defined, so is $\hat{p}$ and $\hat{p} \in\left(\bar{p}, p^{*}\right)$.
} 
Recall from Subsection 3.1 that in the case with perfectly forward looking agents local instability always leads to a stable 2 -cycle. In the case with boundedly rational forward looking agents more complicated dynamics arises. Mathematically, this difference is explained by the fact that in the perfectly forward looking case the map $g_{\infty}$ has two critical points (i.e. points where the map has a local maximum or a local minimum; see Figure 1), whereas in the boundedly rational forward looking case the map $h_{\infty}$ has only one critical point (a local maximum) and the map is linearly decreasing for all $p>p^{*}$. In particular, if the local instability is strong enough (i.e. $b / B$ is large enough), the steady state $p^{*}$ of the map $h_{\infty}$ has homoclinic points (i.e. points whose time path converges to $p^{*}$ both forward and backward in time). As is well known, existence of homoclinic points implies complicated, chaotic dynamical behavior, as illustrated in the time series of Figures 5 and 6 .

From an economic viewpoint the key difference is that boundedly rational agents make errors in their estimation of expected profits. In particular, when the price $p_{t}>p^{*}$ is large, boundedly rational forward looking agents expect a high profit and, therefore, stick to the simple, naive strategy. This profit expectation turns out to be wrong, and the error might become so large that (almost) all agents switch to the rational strategy, pushing prices back (close) to the steady state. This interaction between local instability and global stability generates chaotic price fluctuations when the intensity of choice is high, as in the case of backward looking agents. Nevertheless, the size of such fluctuations remains limited as in the case for perfectly forward looking agents. Therefore, forward looking behavior dampens the amplitude of price fluctuations, whereas bounded rationality might lead to chaotic (small amplitude) fluctuations.

\section{An optimizing representative agent}

Until now we have focussed on a heterogeneous agent framework, where agents switch between two predictors, a costly sophisticated and a cheap simple rule. The aim of this section is to reconcile the heterogeneous agent framework with that of a representative agent optimally choosing from a continuum of expectation rules of different quality. The representative agent outweighs the benefits of a better prediction rule against the costs of information gathering, in the spirit of Simon $(1955,1957)$. Our approach has been inspired by a recent paper by Dudek (2004); see also Evans and Ramey (1992).

Consider a cobweb model with a representative supplier. As before, the production decision at time $t$ depends upon the prediction $p_{t+1}^{e}$ for the price at time $t+1$. The representative supplier can optimally choose among a continuum of forecasting rules, each with different quality and costs. At time $t$, choosing a predictor with quality $q \in[0,1]$ corresponds to buy, at a cost $C(q)$, a signal $v_{t}$ such that

$$
v_{t}= \begin{cases}p_{t+1} & \text { with probability } \\ p_{t} \quad \text { with probability } & 1-q .\end{cases}
$$

The information gathering cost function, $C(q)$, is assumed to be increasing and convex. To optimally choose the quality of the signal, the representative agent computes his expected 
net profit and maximizes it with respect to $q$. In general, a better signal gives a higher gross expected profit at a higher cost. At period $t$, the trade-off between expected profits and information gathering costs sets the optimal predictor quality, $q_{t}^{*}$, which determines the optimal supply $x_{t}$, which, given the demand, determines the realized price and profit at time $t+1$, and so on and so forth. The purpose of this section is to investigate the equilibrium price dynamics generated by this mechanism and to compare it with the heterogeneous agent models of the previous section.

Similarly to Subsection 3.1, we focus on the case of a perfectly forward looking representative agent; that is, the representative agent behaves "as if" he has perfect foresight on expected profit and no systematic errors are made in estimating expected profits. Expected profits of a representative agent choosing the perfect foresight forecast with probability $q$ and the naive forecast with probability $(1-q)$ is then given by:

$$
\pi_{t+1}^{e}(q)=q \pi\left(p_{t+1}, p_{t+1}\right)+(1-q) \pi\left(p_{t+1}, p_{t}\right) .
$$

In this case the fitness measure for using quality $q$ is given by:

$$
U_{t+1}^{e}(q)=\pi_{t+1}^{e}-C(q)
$$

Similarly to the story underlying the managerial perfect foresight equilibrium of Subsection 3.1 , we assume that there is a continuum of managers who offer to sell a predictor of quality $q \in[0,1]$. The managers have perfect foresight on expected profits. The representative agent can not compute the expected profit by himself, but compares the fitnesses announced by the managers without knowing the value of $C(q)$ and, therefore, being unable to derive $p_{t+1}$ from public information.

To optimally choose the quality of the signal, the representative compares the fitness associated with each predictor $q \in[0,1]$. At period $t$, by choosing the predictor, that is, the level of $q$ that grants him the higher fitness, the agent behaves "as if" he is solving:

$$
\operatorname{Argmax}_{q}\left\{U_{t+1}^{e}(q)\right\}=\operatorname{Argmax}_{q}\left\{q \pi\left(p_{t+1}, p_{t+1}\right)+(1-q) \pi\left(p_{t+1}, p_{t}\right)-C(q)\right\} .
$$

When this maximization problem has an interior solution, this is given by the solution of the first order condition:

$$
\pi\left(p_{t+1}, p_{t+1}\right)-\pi\left(p_{t+1}, p_{t}\right)=\frac{b}{2}\left(p_{t+1}-p_{t}\right)^{2}=C^{\prime}(q) .
$$

The specific solution depends on the functional form of $C(q)$. In general, given a solution of the maximization problem at time $t$, which we shall call $q_{t}^{*}$, we can derive the implicit equation that sets the price at time $t+1$. To do that notice that, by the Law of Large Numbers, on average the representative agent produces:

$$
\bar{S}\left(v_{t}\right)=S\left(\bar{v}_{t}\right)=b q_{t}^{*} p_{t+1}+b\left(1-q_{t}^{*}\right) p_{t} .
$$

At time $t+1$ market clearing (implicitly) defines the price $p_{t+1}$ according to:

$$
A-B p_{t+1}=b q_{t}^{*} p_{t+1}+b\left(1-q_{t}^{*}\right) p_{t}
$$


The price is only implicitly defined because the optimal level of $q$ is, in general, a function of $p_{t}$ and $p_{t+1}$; that is, $q_{t}^{*}=q_{t}^{*}\left(p_{t}, p_{t+1}\right)$. In what follows we investigate the equilibrium price dynamics for different functional forms of the information gathering cost function, $C(q)$.

\subsection{Linear cost function}

Consider first the case of a linear information gathering cost function $C(q)$. The following theorem shows that in the linear case, the price dynamics described by (34) exactly corresponds to the price dynamics of the heterogeneous agent model with perfectly forward looking agents in Subsection 3.1 when $\beta=\infty$.

Theorem 9 When the marginal information gathering costs of the representative agent is constant, that is, $C^{\prime}(q)=C$, the system ((33) and (34)) is equivalent to the heterogeneous agent system ((20) and (21)) with $\beta=\infty$. Consequently, when the market is locally unstable the system ((33) and (34)) always converges to a period 2 orbit.

This is the first interesting correspondence between the models: the representative agent model with a linear cost function for information gathering corresponds exactly to the heterogeneous agent model with intensity of choice $\beta=\infty$. This result might be explained by observing two key features: (i) in the limit $\beta \rightarrow \infty$ all agents choose the best predictor, so that the heterogeneous agent model reduces to a single agent model (possibly switching between strategies over time) and (ii) in the case of a linear information gathering function in each time period typically it is optimal for the representative agent to use an extreme signal; that is, to use either $q^{*}=0$ (naive) or $q^{*}=1$ (rational expectations).

\subsection{Nonlinear cost function}

What is the relation between a heterogeneous agent and a representative agent framework in the case of a general, nonlinear information gathering function $C(q)$ ? To answer this question, it is useful to consider an explicit example. Take as cost function:

$$
C(q ; \alpha)=C q^{\alpha}, \quad \alpha>1
$$

where $C(0)=0, C(1)=C, C^{\prime}(0)=0$ and $C^{\prime}(1)=\alpha C$. In this case the maximization problem (32) may have an interior solution. To find it, we solve the first order condition (33) and get:

$$
q_{t}^{*}=\left[\frac{b / 2\left(p_{t+1}-p_{t}\right)^{2}}{\alpha C}\right]^{\frac{1}{\alpha-1}} .
$$

Notice that as long as the cost function is convex $(\alpha>1)$, the optimal level of $q$ is an increasing function of $p_{t+1}$. This turns out to be important for the uniqueness of the 
(a)

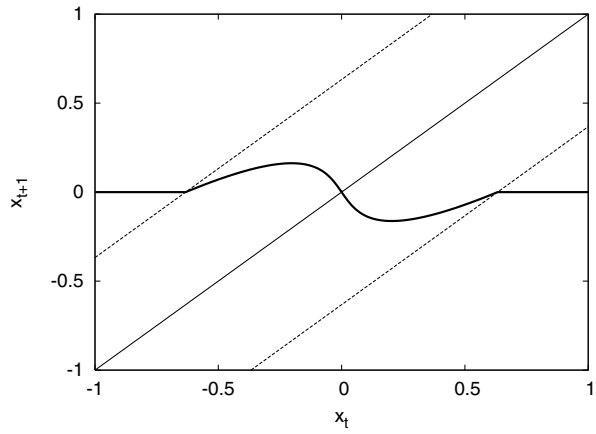

(b)

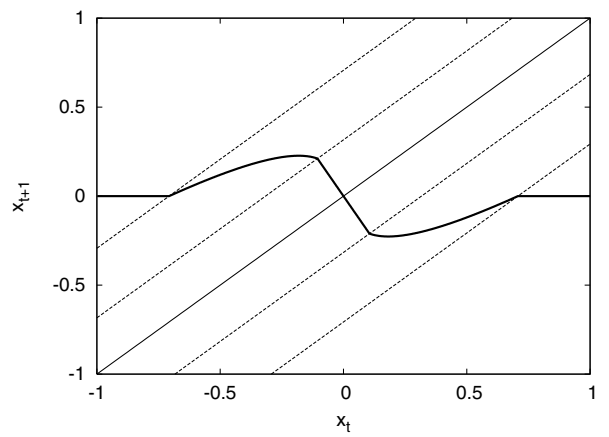

Figure 7 Graphs of the map $x_{t+1}=r\left(x_{t}\right)$ in (37) for different cost functions. (a) $C(q)=0.2 q^{2}$. (b) $C(q)=0.2 q^{2}+0.1 q$. In both cases, due to the symmetry of the map around $x=0$, the dynamics converges to a 2-cycle. The lines parallel to the diagonal delimit the zones where an implicit solution exist, as specified in (37). Other parameters are $B=1$ and $b=2.0$.

market equilibrium price. The market equilibrium equation (34) becomes:

$$
A-B p_{t+1}=\left[\frac{b / 2\left(p_{t+1}-p_{t}\right)^{2}}{\alpha C}\right]^{\frac{1}{\alpha-1}} b\left(p_{t+1}-p_{t}\right)+b p_{t} .
$$

Theorem 10 Let $\alpha>1$. For any fixed value of $p_{t} \geq 0$, the system (35) implies a well defined map $p_{t+1}=r\left(p_{t}\right)$. That is, there exists a unique minimum non-negative price $p_{t+1}$ such that, at time $t+1$, either the market is in equilibrium or the excess supply is positive at $p_{t+1}=0$.

A straightforward calculation shows that for any $\alpha \geq 1$ and $C \geq 0$ the fixed point of the system is $p^{*}=A /(b+B)$, as before. Figure 7 shows a graph of the map in the case of a quadratic cost function $(\alpha=2)$. The following theorem characterizes the price dynamics.

Theorem 11 Let $C(q)=C q^{2}$. When $b / B<1$, the price dynamics converges to the steadystate equilibrium $p^{*}$. When $b / B>1$, the steady state is locally unstable and a unique globally stable 2-cycle exists.

It turns out to be possible to generalize Theorem 11 for any increasing and convex cost function $C(q)$. In fact, the condition for the existence of an interior solution of (32) defines four parallel lines with slope one and intercepts $\sqrt{2 C^{\prime}(1) / b}, \sqrt{2 C^{\prime}(0) / b},-\sqrt{2 C^{\prime}(0) / b}$, and $-\sqrt{2 C^{\prime}(1) / b}$; see the Proof of Theorem 12 for details. We can use these lines to define the price dynamics in different regions, for any given $C(q)$. In some regions delimited by these lines the representative agent chooses either $q=0$ or $q=1$ (see Figure 7). For all other values of $q$ the dynamics is implicitly determined by the solution of the corresponding 
market equilibrium equation. In summary, we have:

$$
p_{t+1}=r\left(p_{t}\right)= \begin{cases}p^{*} & p_{t+1} \geq p_{t}+\sqrt{2 C^{\prime}(1) / b} \\ \text { implicit solution } & p_{t+1} \in\left(p_{t}+\sqrt{2 C^{\prime}(1) / b}, p_{t}+\sqrt{2 C^{\prime}(0) / b}\right) \\ \frac{A}{B}-\frac{b}{B} p_{t} & p_{t+1} \in\left[p_{t}+\sqrt{2 C^{\prime}(0) / b}, p_{t}-\sqrt{2 C^{\prime}(0) / b}\right] . \\ \text { implicit solution } & p_{t+1} \in\left(p_{t}-\sqrt{2 C^{\prime}(0) / b}, p_{t}-\sqrt{2 C^{\prime}(1) / b}\right) \\ p^{*} & p_{t+1} \leq p_{t}-\sqrt{2 C^{\prime}(1) / b}\end{cases}
$$

In terms of the deviation $x_{t}=p_{t}-p^{*}$ from the steady state, the map becomes:

$$
x_{t+1}=r\left(x_{t}\right)= \begin{cases}0 & x_{t} \leq-\sqrt{2 C^{\prime}(1) / b} \\ \text { implicit solution } & x_{t} \in\left(-\sqrt{2 C^{\prime}(1) / b},-\sqrt{2 C^{\prime}(0) / b} \frac{B}{B+b}\right) \\ -\frac{b}{B} x_{t} & x \in\left[-\sqrt{2 C^{\prime}(0) / b} \frac{B}{B+b},+\sqrt{2 C^{\prime}(0) / b} \frac{B}{B+b}\right] . \\ \text { implicit solution } & x_{t} \in\left(+\sqrt{2 C^{\prime}(0) / b} \frac{B}{B+b},+\sqrt{2 C^{\prime}(1) / b}\right) \\ 0 & x_{t} \geq \sqrt{2 C^{\prime}(1) / b}\end{cases}
$$

Figure 7 shows the graph of $r$ in two specific cases. The next theorem shows that only this partial knowledge of the map $r$ is sufficient to characterize the price dynamics:

Theorem 12 Let $C(q)$ be any increasing and convex information gathering cost function. When $b / B<1$ the price dynamics converges to the stable steady-state $p^{*}$; when $b / B>1$ the steady state is locally unstable and a unique globally stable 2-cycle exists.

Notice that according to Theorem 12, in the case of a perfectly forward looking representative agent concerning expected profit, in the long run the incentives to buy a perfect foresight predictor are never strong enough, no matter how strong the local instability of the market is. Stated differently, for any functional form of the cost function, either (when the market is stable) the agent always chooses a cheap naive predictor $(q=0)$ or (when the market is unstable) he switches between a naive predictor and a better, but non-perfect, predictor with $q<1$. Before concluding, we present another interesting relationship between the representative agent and the heterogeneous agent framework of Subsection 3.1.

Theorem 13 There exists a non-decreasing and convex information gathering cost function $C(q)$ such that the price dynamics driven by the interaction of a group of heterogeneous agents choosing between a freely available naive forecast $(q=0)$ and a costly perfect foresight forecast $(q=1)$ at constant cost $C$ as given by (20) and (21) is the same as if a representative agent is operating in the market and optimally chooses a signal $q \in[0,1]$ at cost $C(q)$ as in (33) and (34). For every fixed $\beta$ and $C$ the cost function $C(q)$ is given by:

$$
C(q)=\left\{\begin{array}{ll}
\int_{t_{0}}^{q}\left(\frac{2 \tanh ^{-1}(2 t-1)}{\beta}+C\right) d t, & q \in\left(t_{0}, 1\right] \\
0, & q \in\left[0, t_{0}\right]
\end{array},\right.
$$


where $t_{0}=\left(1+m^{*}\right) / 2$.

It can be shown that this exact correspondence between a heterogeneous agent and a representative agent framework is not only valid in the perfectly forward looking case studied in this section, but holds more generally, for example also in the case of boundedly rational forward looking agents (making mistakes in the estimation of expected profits) or in the original framework of Brock and Hommes (1997) (with backward looking agents and strategy switching based on realized profits). Hence, the same trade-off between local instability and global stability when a group of heterogeneous agent is operating in the market exists when a representative agent optimally chooses the quality of his price predictor. This kind of theorem shows that, for an outside observer, it would be impossible to distinguish between a heterogeneous agent and the corresponding representative agent economy (e.g. the discussion in Kirman (1992)).

\section{Conclusion}

Adaptive rational equilibrium dynamics can produce complicated equilibrium price dynamics, because of the interaction between a locally destabilizing force when agents use simple, cheap strategies and a far from the steady-state globally stabilizing force when errors become so large that most agents switch to the costly rational forecast. In the original framework, strategy selection is driven by experience or regret, as it is based upon a measure of past realized profits. In this paper we investigated how forward looking behavior in strategy selection might affect the co-evolving equilibrium dynamics. With forward looking behavior, the same local instability as a result of costly information gathering and free riding remains, but the amplitude of price fluctuations is dampened. How exactly forward looking behavior affects the globally stabilizing force depends upon how sophisticated agents are in computing expected profits. When agents are able to make a perfect forecast for expected profits, prices lock into a stable 2-cycle and the errors of the cheap naive strategy remain small enough for the population of agents never to switch completely to the costly rational forecast. If, however, agents can only make a boundedly rational forecast of expected profits, errors might grow big enough for (almost) all agents to switch to the costly rational strategy. In that case, the globally stabilizing force becomes strong enough to push prices back close to the (locally unstable) steady state, therefore causing irregular switching between price fluctuations of low and moderate amplitude. Boundedly rational forward looking agents thus dampen the amplitude of the price oscillations but a rational route to randomness, as in the backward looking case, remains.

We have also established an equivalence relation between heterogeneous agent models with evolutionary switching of strategies and a representative agent who optimally chooses between the benefits of high quality forecasts and the associated information gathering costs. To an outside observer it is impossible to distinguish between the heterogeneous agent model and the corresponding optimal representative agent framework.

We emphasize that we have shown these results to hold in a supply driven commodity market, with negative expectational feedback (i.e. a high price forecast leads to high 
production and, therefore, a low realized market price). An interesting question for future work is whether similar results hold for speculative asset markets. In fact, for asset markets we conjecture that forward looking behavior might actually destabilize the amplitude of price fluctuations, because of the positive expectational feedback (i.e. high expectations of future asset prices lead to increased asset demand and thus higher realized market prices). We leave this conjecture for future research.

Another important topic for future research is how "invasion" of boundedly rational strategies affects the long-run outcome of a market with heterogeneous agents. In our cobweb framework, the answer at least depends whether or not more memory comes at higher costs. When there are no costs, invasion of boundedly rational strategies detecting and exploiting the regular, periodic structure might stabilize the adaptive equilibrium dynamics. However, when memory comes at a higher cost we have seen an example where a stable 2-cycle is persistent against invasion of a period-2 forecasting rule into a market with free naive versus costly rational expectations. Because of its lower costs, the period-2 rule drives out most of the rational agents, but the stable 2-cycle persists with smaller amplitude. Because the period-2 forecasting rule is optimal (it coincides with perfect foresight) with minimal use of memory, it seems that this situation is "evolutionary stable" and persistent against invasion of other boundedly rational types. Another possibly "evolutionary stable" or persistent long-run outcome might be the situation where the interaction between more and more boundedly rational strategies leads to chaotic small amplitude price oscillations whose structure can not be detected and exploited easily by newly invading boundedly rational strategies. A theory of "evolutionary stable" long-run outcomes with complicated, irregular equilibrium market dynamics is an important topic for a future research agenda.

\section{Appendix}

\subsection{Proofs of Section 2}

Proof of Lemma 1: The map $f_{\infty}$ defined in (10) is two-dimensional. Define $f_{\infty}^{1}$ as a one-dimensional projection of $f_{\infty}$ according to

$$
f_{\infty}^{1}\left(p_{t}\right)=f_{\infty}\left(p_{t},\left(f_{\infty, 1}^{-}\right)^{-1}\left(p_{t}\right)\right)
$$

where $f_{\infty, 1}^{-}(\cdot)=F_{\infty, 1}(\cdot,-1)$ and $F_{\infty, 1}$ is the first component of the map $F_{\beta}$, for $\beta=\infty$, defined in Section 2 below equations (8) and (9). By applying this definition, one can easily show that

$$
f_{\infty}^{1}\left(p_{t}\right)= \begin{cases}\frac{A}{B}-\frac{b}{B} p_{t}, & \text { if } p_{t} \in\left[p^{*}-\varepsilon, p^{*}+\varepsilon\right], \\ p^{*}, & \text { otherwise }\end{cases}
$$

where $\varepsilon=\frac{b}{b+B} \sqrt{2 C / b}$. The graph of $f_{\infty}^{1}$ is given in Figure 2. The lemma follows by noticing that the difference between the maximum and the minimum of the map $f_{\infty}^{1}$ is $2 \frac{b^{2}}{B(B+b)} \sqrt{2 C / b}$.

\subsection{Proofs of Section 3}

Proof of Theorem 3: From (20) the excess supply function $E S\left(p_{t}, p_{t+1}\right)$ is given by:

$$
E S\left(p_{t}, p_{t+1}\right)=\frac{b}{2}\left(p_{t+1}\left(1+m\left(p_{t}, p_{t+1}\right)\right)+p_{t}\left(1-m\left(p_{t}, p_{t+1}\right)\right)\right)-A+B p_{t+1},
$$


where

$$
m\left(p_{t}, p_{t+1}\right)=m_{t}=\tanh \left(\frac{\beta}{2}\left[\frac{b}{2}\left(p_{t+1}-p_{t}\right)^{2}-C\right]\right) .
$$

Consider for a moment the function $E S\left(p_{t}, y\right)$. We define a function $y=\tilde{g}\left(p_{t}\right)$ such that $E S\left(p_{t}, y\right)=0$. A straightforward computation shows that:

$$
\frac{\partial E S\left(p_{t}, y\right)}{\partial y}=\frac{b}{2}\left(1+m_{t}+\beta \frac{b}{2}\left(y-p_{t}\right)^{2}\left(1-m_{t}\right)\left(1+m_{t}\right)\right)+B>0 .
$$

This implies that given any value of $p_{t}$ we can always find a unique $y$ such that $E S\left(p_{t}, y\right)=0$. Consequently, the function $y=\tilde{g}\left(p_{t}\right)$ is well defined and, by the implicit function theorem, the function $\tilde{g}$ is $C^{1}$. Define the map $g_{\beta}$ as follows:

$$
g_{\beta}\left(p_{t}\right)=\left\{\begin{array}{lll}
\tilde{g}\left(p_{t}\right) & \text { if } & \tilde{g}\left(p_{t}\right) \geq 0 \\
0 & \text { if } & \tilde{g}\left(p_{t}\right)<0
\end{array}\right.
$$

This means that when $g_{\beta}\left(p_{t}\right)=p_{t+1}>0$, we have $E S\left(p_{t}, p_{t+1}\right)=0$, whereas when $g_{\beta}\left(p_{t}\right)=p_{t+1}=0$, there is excess supply even when the market price is $p_{t+1}=0$. In fact, $\partial E S\left(p_{t}, y\right) / \partial y>0$ implies that when $\tilde{g}\left(p_{t}\right)<0, E S\left(p_{t}, 0\right)>E S\left(p_{t}, \tilde{g}\left(p_{t}\right)\right)=0$. Finally, the map $g$ is continuous but in general not differentiable at the lowest price $p_{t}$ for which $g_{\beta}\left(p_{t}\right)=0$.

Proof of Theorem 4: $\quad$ In terms of $x_{t}=p_{t}-p^{*}$ the system (20-21) becomes:

$$
\left\{\begin{array}{l}
-B x_{t+1}=\frac{b}{2}\left(x_{t+1}\left(1+m_{t}\right)+x_{t}\left(1-m_{t}\right)\right) \\
m_{t}=\tanh \left(\frac{\beta}{2}\left[\frac{b}{2}\left(x_{t+1}-x_{t}\right)^{2}-C\right]\right)
\end{array}\right.
$$

with fixed point $x^{*}=0$ and corresponding $m^{*}=\tanh (-\beta C / 2)$. Similarly, as in the proof of Theorem 3 it follows that there exists a well defined map $x_{t+1}=\bar{g}_{\beta}\left(x_{t}\right)$, obtained from the map $p_{t+1}=g_{\beta}\left(p_{t}\right)$ by choosing $p^{*}$ as the origin. To keep the notation simple we drop the bar, and write $g$ instead of $\bar{g}$ in what follows. The following properties of the map $g$ will be useful to prove the Theorem:

(a) $g$ is odd; that is, $g(-x)=-g(x)$, and $g(x)>0$ when $x<0$.

(b) When $\beta>\beta_{1}$ there exists a unique period 2 orbit $\{a,-a\}$, satisfying $g(g(a))=a$ and $g(a)=-a$.

(c) $g^{\prime}(0)<g^{\prime}(x)<1$, for all $x$.

(d) The map $g$ has two critical points, $c$ and $-c$. Furthermore, $g^{\prime}(x)<0$ iff $x \in(-c, c)$, so that $g$ has a local minimum at $x=c$ and a local maximum at $x=-c$.

We postpone the proof of properties $(a)-(d)$, and first use them to prove (i)-(iii) in Theorem 4 . Notice that $(a)$ and $(d)$ imply that $g(c)$ is in fact a global minimum and $g(-c)$ a global maximum. Using the implicit function theorem, we get:

$$
g^{\prime}(x)=-\left.\frac{\frac{\partial E S(x, y)}{\partial x}}{\frac{\partial E S(x, y)}{\partial y}}\right|_{y=g(x)}=\left.\frac{-b(1-\alpha)(1-m(x, y))}{b(1+m(x, y)+\alpha(1-m(x, y)))+2 B}\right|_{y=g(x)},
$$

where

$$
\alpha=\frac{b}{2} \beta(y-x)^{2}(1+m(x, y))
$$


(a)

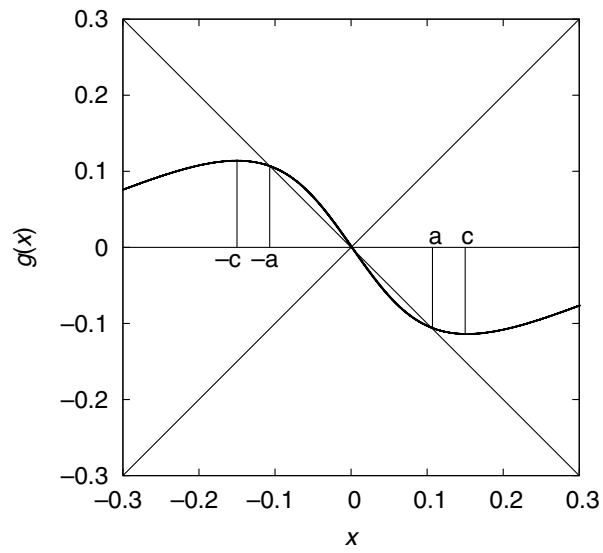

(b)

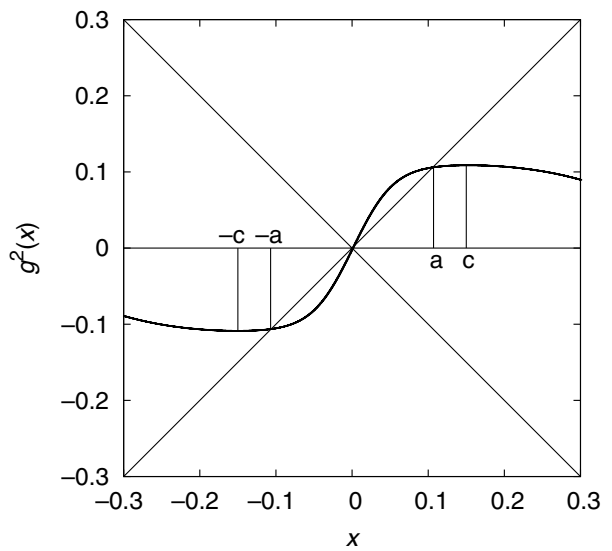

Figure 8 Graph of (a) the map $g_{\infty}$ and (b) its second iterate $g^{2}$ in the case when $c>a$.

$$
A=1.5, B=1, b=2, C=0.1 \text { and } \beta=20 \text {. }
$$

$E S(x, y)$ is the excess supply function defined in $(38)$ and $m(x, y)$ is the difference of agents' fractions in (39).

ProOf OF ( $i$ ): The global stability of $x^{*}=0$ when $C=0$ follows from the fact that in this case $g$ is a contraction. Indeed, using (40) one gets $g^{\prime}(0)=-b /(b+2 B)>-1$, so that property $(c)$ implies $g^{\prime}(x) \in(-1,1)$ for all $x$.

ProOF OF (ii): The global stability of the fixed point when $\beta<\beta_{1}$ follows again from the fact that $g$ is a contraction. In fact,

$$
g^{\prime}(0)=\lambda(\beta)=-\frac{b(1-m(0,0))}{b(1+m(0,0))+2 B}>-1, \quad \text { iff } \quad \beta<\beta_{1},
$$

so that $(c)$ implies $g^{\prime}(x) \in(-1,1)$. Local instability follows from the fact that $g^{\prime}(0)<-1$ when $\beta>\beta_{1}$. The value of $\beta_{1}$ is determined by the condition $g^{\prime}(0)=-1$, which gives $m(0,0)=m^{*}=-B / b$.

ProOf OF ( $i i i)$ : Uniqueness of the period 2 cycle follows from $(b)$. To show global stability of the 2-cycle we have to characterize the shape of the map $g^{2}$. By $(d)$ the map $g$ has only two critical points, $-c<0$ (local maximum) and $c>0$ (local minimum). From now on we concentrate on $g^{2}$ for $x>0$; the results for $x<0$ follow by symmetry. We look for the critical points of $g^{2}$; that is, for the points where $\left(g^{2}\right)^{\prime}(x)=g^{\prime}(g(x)) g^{\prime}(x)=0$. The positive critical points of $g^{2}$ are the positive critical point $c$ of $g$, and points $d>0$ such that $g(d)=-c$. We distinguish two cases.

Case 1: $g(c)>-c$ and $g(-c)<c$ (see Figure 8). In this case, because $c$ is larger than the global maximum $g(-c)$, there is no $d>0$ such that $g(-d)=c$. Hence, $c$ and $-c$ are the unique critical points of $g^{2}$. We claim that $\left(g^{2}\right)^{\prime}(x)>0$ when $x \in(-c, c)$. This follows because $x \in(-c, c)$ implies both $g^{\prime}(x)<0$ and $g(x) \in(-c, c)$ so that $\left(g^{2}\right)^{\prime}(x)=g^{\prime}(g(x)) g^{\prime}(x)>0$. This, together with $g(c)>-c$, implies that at the unique point $a>0$ for which $g(a)=-a$, we have $a<c$ and $g^{\prime}(a)>0$. When $\beta>\beta_{1}, g^{\prime}(0)<-1$, so that $\left(g^{2}\right)^{\prime}(0)>1$. Hence, $x=a$ is the unique intersection point of $g^{2}(x)$ with $y=x$ and $\left(g^{2}\right)^{\prime}(a)<1 .\{a,-a\}$ is therefore a locally stable 2-cycle. Because $g^{2}(x)>0$ for all $x>0$ all points (except the unstable steady state) converge and the 2-cycle is globally stable.

Case 2: $\quad g(c)<-c$ and $g(-c)>c$ (see Figure 9). In this case there exist two other positive critical points of $g^{2}, d_{1}$ and $d_{2}, d_{1}<c<d_{2}$, for which $g(x)=-c$. Moreover, property $(b)$ implies that $a$ is the unique positive 
(a)

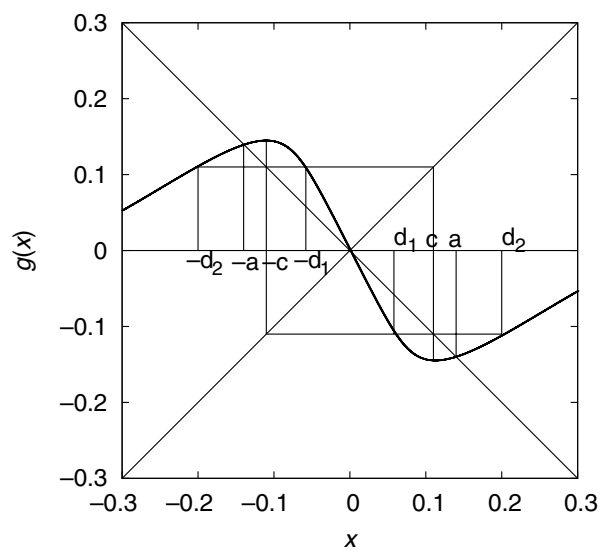

(b)

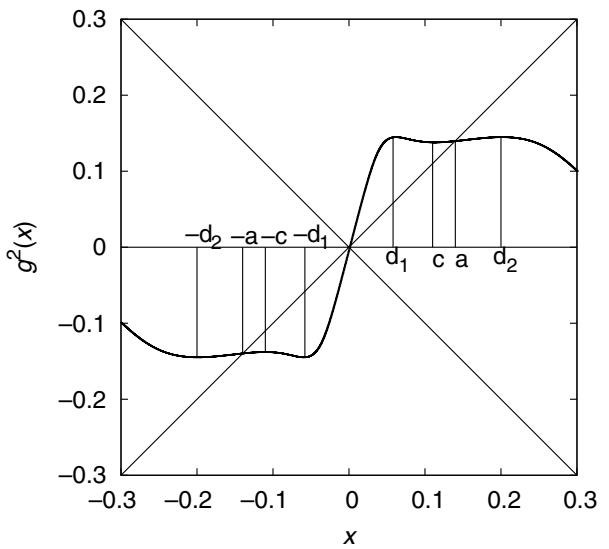

Figure 9 Graph of (a) the map $g_{\infty}$ and (b) its second iterate $g^{2}$ in the case when $c<a$. $A=1.5, B=1, b=2, C=0.1$ and $\beta=50$.

intersection point of $g(x)$ with the line $y=-x$, and because $g(c)<-c$ we have $d_{1}<c<a<d_{2}$. By symmetry, $-d_{1}$ and $-d_{2}$ are also critical points of $g^{2}$ and $-d_{2}<-a<-c<d_{1}$. Clearly, $g^{2}\left(d_{1}\right)$ and $g^{2}\left(d_{2}\right)$ are local maxima and $g^{2}(c)$ is a local minimum, and using (c) and (d) we get $0 \leq\left(g^{2}\right)^{\prime}(x)<1$, for all $x \in\left[c, d_{2}\right]$. This implies that $a \in\left(c, d_{2}\right)$ is a locally stable fixed point of $g^{2}$. From the global shape of the graph of $g^{2}$ it follows easily that the 2-cycle is globally stable.

To conclude the proof we have to show that $(a),(b),(c)$ and $(d)$ hold.

(a) In deviations $x$ from the steady state the excess supply function is

$$
E S\left(x_{t}, x_{t+1}\right)=\frac{b}{2}\left(x_{t+1}\left(1+m\left(x_{t}, x_{t+1}\right)\right)+x_{t}\left(1-m\left(x_{t}, x_{t+1}\right)\right)\right)+B p_{t+1} .
$$

If $E S(x, g(x))=0$ then also $E S(-x,-g(x))=0$, implying $g(-x)=-g(x)$. Moreover, when $x>0(x<0)$ the only possibility to have $E S(x, g(x))=0$ is $g(x)<0(g(x)<0)$.

(b). We are looking for a point $a$ such that $g(g(a))=a$. If such a point exists, $E S(a, g(a))=0$ implies $E S(g(a), g(g(a)))=E S(g(a), a)=0$ and vice versa. Hence, the existence and uniqueness of a period-2 orbit $\{a, g(a)\}$ is equivalent to the existence and uniqueness of $a$ such that $E S(a, g(a))=E S(g(a), a)=0$. Consider the change of variable $z=a+g(a), w=g(a)-a$. We can rewrite $E S(a, g(a))=E S((z-w) / 2,(z+w) / 2)=$ $\widetilde{E S}(w, z)$. In terms of the new variables and the function $\widetilde{E S}$, the existence and uniqueness of $a$ is equivalent to the existence and uniqueness of two points $\bar{z}$ and $\bar{w}$ such that both $\widetilde{E S}(\bar{w}, \bar{z})=\widetilde{E S}(-\bar{w}, \bar{z})=0$. Writing down these two conditions as a function of the new variables one obtains:

$$
\left\{\begin{array}{l}
\bar{z}=-\frac{B+b m(\bar{w})}{B+b} \bar{w} \\
\bar{z}=\frac{B+b m(\bar{w})}{B+b} \bar{w}
\end{array}\right.
$$

where, with abuse of notation, $m(w)$ is the difference of fraction in (39) defined as a function of $g(a)-a=w$. Notice that if a solution of (42) exists, then $\bar{z}=0$ and $b m(\bar{w})=-B$. Obviously $\bar{z}=0$ implies $g(a)=-a$. The other equation $b m(\bar{w})=-B$ is only possible when $\beta>\beta_{1}$. In fact, when $\beta<\beta_{1}, m(0)>-B / b$ so that 
$m(w)>m(0)$ implies $m(w)>-B / b$. When $\beta>\beta_{1}, b m(w)=-B$ has two symmetric solutions. Consequently, a unique symmetric 2 -cycle $\{a,-a\}$ exists for $\beta>\beta_{1}$.

(c). The expression of $g^{\prime}(x)$ in (40) can be rewritten as:

$$
g^{\prime}(x)=\frac{-b(1-\alpha)}{b\left(\frac{(1+m(x, y))}{(1-m(x, y))}+\alpha\right)+\frac{2 B}{(1-m(x, y))}},
$$

where

$$
\alpha=\frac{b}{2} \beta(y-x)^{2}(1+m(x, y))
$$

Because the difference of fractions $m$ is an increasing function of $(y-x)^{2}, \alpha$ is also an increasing function of $(y-x)^{2}$. Moreover, $\alpha \geq 0$ and $\alpha=0$ when $(y-x)^{2}=0$. These properties together with $m \in(-1,1)$ imply that the denominator of $g^{\prime}(x)$ is a positive increasing function of $(y-x)^{2}$ and that the numerator is an increasing function of $(y-x)^{2}$, which is negative when $(y-x)^{2}=0$. These facts imply that the minimum of $g^{\prime}(x)$ is achieved when $y-x=0$; that is, when $y=x$, which implies $x=0$. Moreover, when $g^{\prime}(x)>0$, that is, when $\alpha>1$, one can easily show that the numerator is always smaller then the denominator so that $g^{\prime}(x)<1$ for all $x$.

(d) A critical point $c$ satisfies $g^{\prime}(c)=0$. From (43), and from the fact that $\alpha$ is increasing in $(y-x)^{2}$ and $\alpha=0$ for $(y-x)^{2}=0$, it follows that there exists a unique $(y-x)^{2}=k$ such that $g^{\prime}=0$. To show that $g$ has a unique critical point $c>0$, we have to show that $c$ is the unique solution of $(g(c)-c)^{2}=k$. This translates into showing that $g(c)-c=-\sqrt{k}$ has a unique positive solution. ${ }^{5}$ We claim here the more general statement that the line $y=x-h$ and the curve $y=g(x)$ intersect only once when $x>0, \forall h>0$. Change variables from $(x, y)$ to $(x, h=x-y)$, and consider $E S$ as a function of the new variables. We have $E S(x, y)=E S(x, x-h)=\widehat{E S}(x, h)$. One can easily show that this holds:

$$
\frac{\partial \widehat{E S}(x, h)}{\partial x}=\frac{\partial(b / 2((1+m(h))(x-h)+(1-m(h)) x)+B(x-h))}{\partial x}=B+b>0 .
$$

As a consequence, we can apply the implicit function theorem and find function $t$ such that $\widehat{E S}(x=t(h), h)=0$. This means that for $\forall h>0$ there exists a unique $x=t(h)$ and, as a consequence, a unique $y=x-h$ where $\widehat{E S}(x, h)=\widehat{E S}(x, x-y)=E S(x, y)=0$. From the last expression it follows that $y=g(x)$, so that, for every given $h$, there exists a unique point $(x, g(x))$ with $g(x)=x-h$. From this we conclude that $g(x)-x=-\sqrt{k}$ has a unique solution $c$. Property $(a)$ implies $c>0$. Because $c$ is the unique positive critical point, by symmetry $-c$ is also a critical point. Because $c$ and $-c$ use the only critical points and $g^{\prime}(0)<0$ it must be that $g^{\prime}(x)<0$ iff $x \in(-c, c)$. This implies that $g(c)$ is a local minimum and $g(-c)$ a local maximum.

Proof of Theorem 5: The existence of a 2-cycle can be proven along the same lines as in Theorem 4. Consider the explicit definition of $g_{\infty}$ in (22). Let $c$ and $-c$ be the critical points and $d_{2}>c$ and $-d_{2}<-c$ points such that $g\left(d_{2}\right)=-c$ and $g\left(-d_{2}\right)=c$ as in the proof of Theorem 4. A straightforward computation shows that $g^{2}(x)=x$ for all $x \in\left[-d_{2}, c\right] \cup\left[c, d_{2}\right]$. This implies that $g$ has a continuum of 2-cycles. The computation of $\Delta_{\infty}^{g}$ follows directly from the definition of the map $g_{\infty}$ in (22).

Proof of TheOrem 5: From (25) excess supply is given by

$$
E S\left(p_{t}, p_{t+1}\right)=\frac{b}{2}\left[p_{t+1}\left(1+m_{t}\right)+p_{t}\left(1-m_{t}\right)\right]-A+B p_{t+1}=0,
$$

where, as in (26),

$$
m_{t}=\tanh \left(\frac{\beta}{2}\left[\frac{b}{2}\left(p_{t+1}^{2}-p_{t}^{2}\right)-C\right]\right)
$$

\footnotetext{
${ }^{5}$ Because by $(a) g(x)<0$ when $x>0$, in this case the equation $g(c)-c=+\sqrt{k}$ has no positive solution.
} 
We are looking for a function $h$ (we drop the subscript $\beta$ here) such that $E S\left(p_{t}, h\left(p_{t}\right)\right)=0$. First, we show that there always exists a unique minimum non-negative price $p_{t+1}$ such that, at time $t+1$, either the market is in equilibrium or the excess supply is positive at $p_{t+1}=0$. Second, we show that under certain parameter restrictions $h$ is continuous.

Existence. Write $y=p_{t+1}$ and $x=p_{t}$, and let $p^{*}=A /(B+b)$ as usual and notice that $E S\left(p^{*}, p^{*}\right)=0$. Consider $y>x$; it can be verified that in this case:

$$
\frac{\partial E S(x, y)}{\partial y}=\frac{b}{2}\left(1+m_{t}+\frac{\beta}{2} b y(y-x)\left(1-m_{t}\right)\left(1+m_{t}\right)\right)+B>0 .
$$

Consequently, we can apply the implicit function theorem and there exists a $C^{1}$ map $h$ such that $E S(x, h(x))=0$. The case $y<x$ is more difficult, because $\partial E S(x, y) / \partial y$ might be zero. We can rewrite the condition $E S(x, y)=0$ as:

$$
\frac{2(A-(B+b) y)}{b(y-x)}=m(y, x)-1
$$

where

$$
m(y, x)=\tanh \left(\frac{\beta}{2} \frac{b}{2}\left(y^{2}-x^{2}\right)-\frac{\beta}{2} C\right) .
$$

For $y<x,-2<m-1<-1$ together with (45) imply

$$
\frac{A}{B}-\frac{b}{B} x<y<\frac{2 A}{2 B+b}-\frac{b}{2 B+b} x
$$

and, therefore, also $x>p^{*}$. Equation (45) and $-2<m-1<-1$ also imply that when $y=0$ there is a value $\tilde{x} \in(A / b, 2 A / b)$ such that $E S(\tilde{x}, 0)=0$. Furthermore, because

$$
\left.\frac{\partial E S(x, y)}{\partial x}\right|_{y=0}=\left.\frac{b}{2}\left(1-m_{t}-\beta b x(y-x)\left(1-m_{t}\right)\left(1+m_{t}\right)\right)\right|_{y=0}>0
$$

such a value is unique and $E S(x, 0)>0$ for $x>\tilde{x}$, so that we can define $h(x)=0$ for $x \in[\tilde{x}, \infty)$. Notice that for all $y \leq x, \partial E S(x, y) / \partial x>0$. By the implicit function theorem there exists a $C^{1}$ function $x=s(y)$ such that $E S(s(y), y)=0$. Clearly, $s\left(p^{*}\right)=p^{*}$ and $s(0)=\tilde{x}$. Furthermore, by $(46), s(y)$ must always be between the lines $y=A / b-b / B x$ and $y=2 A /(2 B+b)-b /(2 B+b) x$. The function $h$ we are looking for is not well defined yet because many different $y$ values may be mapped to the same $x$ through the map $s$. However, when this is the case, we can always choose the minimum of these $y$ values. Consequently, there always exists a unique minimum non-negative price $y=p_{t+1}$ such that, at time $t+1$, either the market is in equilibrium or the excess supply is positive at $p_{t+1}=0$ so that $h$ is well defined. Notice that $y=p_{t+1}$ is the lowest non-negative price for which $E S\left(p_{t}, y\right) \geq 0$.

CONTINUITY. The map $h$ defined above may be discontinuous. A sufficient condition for continuity can be obtained by restricting parameters values such that $\partial E S(x, y) / \partial y>0$, also for $y<x$. To obtain such restriction we use (44) and (45) to evaluate $\partial E S(x, y) / \partial y>0$ in those points $(x, y)$ for which $E S(x, y)=0$ :

$$
\left.\frac{\partial E S(x, y)}{\partial y}\right|_{E S=0}=B+(1+m(x, y)) \frac{b}{2}(1-\beta y(A-(B+b) y))>0 .
$$

Because (46) implies that $0<y<p^{*}$, we have $1-\beta y(A-(B+b) y)$ has a minimum value $1-\left(\beta A p^{*} / 4\right)$. As a consequence, condition (48) is satisfied when

$$
B+(1+m(x, y)) \frac{b}{2}\left(1-\beta \frac{A p^{*}}{4}\right)>0 .
$$


(a)

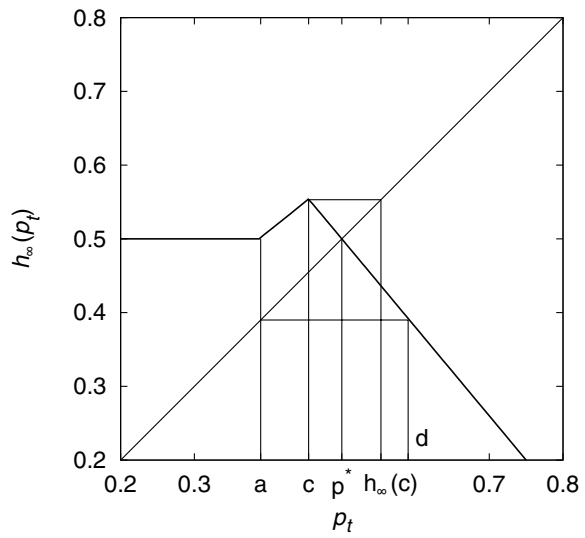

(b)

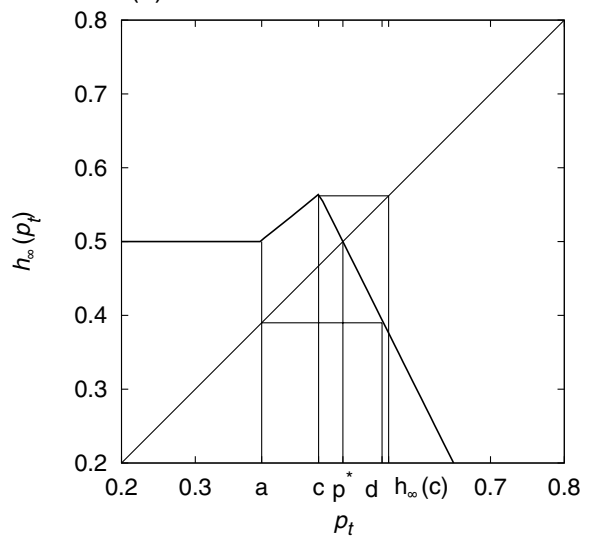

Figure 10 Graphs of the map $h_{\infty}$. In (a) $(b / B=1.2) h_{\infty}(c)<d$ implies that the price fluctuations remain bounded away from $p^{*}$ in the long run. In (b), $(b / B=2), h_{\infty}(c)>d$ implies that the map has (infinitely many) homoclinic points and a typical price time series converges to the locally unstable steady state $p^{*}$. Other parameters: $A=1.5, b=2$ and $C=0.1$.

This is clearly always the case when $\beta<4 /\left(A p^{*}\right)$. Otherwise, when $\beta \geq 4 /\left(A p^{*}\right)$, because $0 \leq y<x$, condition (49) is satisfied when

$$
B+(1+m(0,0)) \frac{b}{2}\left(1-\beta \frac{A p^{*}}{4}\right)>0
$$

which can be rewritten as condition (27) in Theorem 6.

Proof of Theorem 7: From Theorem 5 one can derive that the map $h_{\beta}(p)$ is always well defined and differentiable in a neighborhood of the point $\left(p^{*}, p^{*}\right)$. Furthermore, one can use expressions (44) and (47) to compute:

$$
\left(h_{\beta}\right)^{\prime}\left(p^{*}\right)=\lambda(\beta)=-\frac{\left.(\partial E S(x, y) / \partial x)\right|_{\left(p^{*}, p^{*}\right)}}{\left.(\partial E S(x, y) / \partial y)\right|_{\left(p^{*}, p^{*}\right)}}=-\frac{b\left(1-m^{*}(\beta)\right)}{2 B+b\left(1+m^{*}(\beta)\right)} .
$$

The value of $\beta_{1}$ is found by imposing $\lambda(\beta)=-1$. Local stability follows from $\lambda(\beta) \in(-1,0)$ when $\beta<\beta_{1}$. When $\beta>\beta_{1}, \lambda(\beta)<-1$ and graphical analysis of $h_{\beta}^{2}$ shows that $h_{\beta}^{2}$ has (at least) two other intersection points with the diagonal than $p^{*}$.

Proof of Theorem 8: Consider the map $h_{\infty}$ given in (28). Call $\bar{p}=a$ and $\hat{p}=c$; that is, $a$ solves $\sqrt{a^{2}+2 C / b}=$ $p^{*}$ and $c$ solves $A / B-(b / B) c=\sqrt{c^{2}+2 C / b}$. One can easily show that these points always exist, as long as $2 C / b<\left(p^{*}\right)^{2}$, which we assume here, are unique, and $c>a$. In terms of the points $a$ and $c$, the map $h_{\infty}$ can be rewritten as:

$$
h_{\infty}\left(p_{t}\right)= \begin{cases}p^{*}, & 0 \leq p_{t}<a \\ \sqrt{p_{t}^{2}+2 C / b}, & p \in[a, c] \\ p^{*}-(b / B)\left(p_{t}-p^{*}\right), & p_{t}>c .\end{cases}
$$

The map $h_{\infty}$ has a global maximum at the critical point $c$. Let $d$ be the point such that $h_{\infty}(d)=a$. Notice that when $p>d, h_{\infty}^{2}(p)=p^{*}$. Two examples of the map are given in Figure 10. 
Because $b / B>1$, the steady state $p^{*}$ is locally unstable. In what follows we show that for $b / B$ sufficiently large, there are (infinitely many) homoclinic points, whose orbits converge to $p^{*}$ both forward and backward in time. We consider two cases:

Case 1: $\quad h_{\infty}(c)<d$ (as in Figure 10(a)). When $h_{\infty}(c)<d$ we have $h_{\infty}^{2}(c)<h_{\infty}^{4}(c)<p^{*}<h_{\infty}^{3}(c)<h_{\infty}(c)$. Let $I_{1}=\left[h_{\infty}^{2}(c), h_{\infty}^{4}(c)\right]$ and $I_{2}=\left[h_{\infty}^{3}(c), h_{\infty}(c)\right]$. In this case it is easy to show that all initial states $p_{0}$ are "repelled" from $p^{*}$ and mapped in $I_{1} \cup I_{2}$ after some iterations. Therefore, the long-run dynamics is contained in the set $I_{1} \cup I_{2}$ and bounded away from $p^{*}$.

Case 2: $\quad h_{\infty}(c) \geq d$ (as in Figure 10(b)). In this case the critical point $c$ is a homoclinic point. It converges to $p^{*}$ forward in time and also backwards in time (take successive inverse images $\mathrm{x}>\mathrm{c}$, with $h_{\infty}^{k}(x)=c, k=1,2, \ldots$ ). In fact, there is an interval $I$ of homoclinic points containing $c$.

To conclude the proof we have to show that there exists an $M>1$ such that when $b / B>M$ then case 2 obtains; that is, $h_{\infty}(c) \geq d$. We derive the existence of such a value $M$ from the dependence of the point $d$ and $h_{\infty}(c)$ upon $b / B$. Define $B / b=N, N \in(0,1)$. Depending upon $N, p^{*}, C$ and $b$, the point $d$ and the image $h_{\infty}(c)$ are defined as:

$$
\begin{aligned}
& d(N)=p^{*}+N\left(p^{*}-\sqrt{\left(p^{*}\right)^{2}-2 C / b}\right) \\
& h_{\infty}(c ; N)=p^{*}+\frac{p^{*}}{1-N}\left(\sqrt{1+\frac{1-N}{1+N}(2 C) / b}-1\right) .
\end{aligned}
$$

One can show that $d(0)<h_{\infty}(c ; 0), d(1)>h_{\infty}(c ; 1)$ and $d^{\prime}(N)>0$. Furthermore, $\left(p^{*}\right)^{2}>2 C / b$, which is assumed here to guarantee the existence of $a$ and $c$, is a sufficient condition for $\partial h_{\infty}(c ; N) / \partial N<0$. These facts imply that there exists a unique $N$ such that $d(N)=h_{\infty}(c ; N)$. It follows that there exists a unique $M=1 / N$ such that: $(i)$ when $b / B \in(1, M]$, Case 1 applies, and (ii) when $b / B>M$, Case 2 applies.

\subsection{Proofs of Section 4}

Proof of Theorem 9: When $C^{\prime}(q)=C$ the first order condition (33) for the optimal choice of $q$ becomes:

$$
\Delta \pi_{t+1}=\frac{b}{2}\left(p_{t+1}-p_{t}\right)^{2}=C
$$

Consequently, in general there is no interior solution, but $q_{t}^{*}=1$ if $\Delta \pi_{t+1}>C$ and $q_{t}^{*}=0$ if $\Delta \pi_{t+1}<C$. This implies that the equilibrium price dynamics (34) is governed either by naive expectations or by rational expectation, which gives exactly the same dynamical system as in (20)-(21) with $\beta=+\infty$, or equivalently as in (22).

Proof of Theorem 10: Consider the market equilibrium equation (35):

$$
A-B p_{t+1}=\left[\frac{b / 2\left(p_{t+1}-p_{t}\right)^{2}}{\alpha C}\right]^{\frac{1}{\alpha-1}} b\left(p_{t+1}-p_{t}\right)+b p_{t} .
$$

Notice that, given the values of the parameters $A, B, b, \alpha, C$, for any fixed value of $p_{t} \geq 0$ the demand (left hand side) is a decreasing function of $p_{t+1}$, whereas the supply (right hand side) is an increasing function of $p_{t+1}$ provided that $\alpha>1$. This implies that there exists a unique point $x \in \mathcal{R}$ where demand and supply are equal. Notice that this point is negative for those values of $p_{t}$ for which there is excess supply at $p_{t+1}=0$; that is, when

$$
\left(1-\left[\frac{b p_{t}^{2}}{2 \alpha C}\right]^{\frac{1}{\alpha-1}}\right) b p_{t} \geq A
$$


In this case we set $p_{t+1}=0$, and otherwise $p_{t+1}=x$.

Proof of TheOrem 11: This is just a special case of Theorem 12.

Proof of Theorem 12: Market clearing in (34) implicitly defines a map $p_{t+1}=r\left(p_{t}\right)$ by $E S\left(p_{t}, r\left(p_{t}\right)\right)=0$; that is,

$$
E S\left(p_{t}, r\left(p_{t}\right)\right)=q_{t}^{*} b\left(r\left(p_{t}\right)-p_{t}\right)+b p_{t}-B p_{t+1}+A=0
$$

where $q_{t}^{*}$ solves the maximization problem (32), whose first order condition is

$$
\Delta \pi_{t+1}=\frac{b}{2}\left(p_{t+1}-p_{t}\right)^{2}=C^{\prime}(q)
$$

The first order condition (51) gives only the interior optimal solution. From the convexity of $C(q)$ it follows that $C^{\prime}(0)$ is a global minimum and $C^{\prime}(1)$ is a global maximum of $C^{\prime}(q)$. Hence, $q_{t}^{*}=0$ if $\Delta \pi_{t+1} \leq C^{\prime}(0)$ and $q_{t}^{*}=1$ if $\Delta \pi \geq C^{\prime}(1)$. These two conditions on $\Delta \pi_{t+1}$ define four parallel lines in the plane $\left(p_{t}, p_{t+1}\right)$ with slope 1 and intercepts $\sqrt{2 C^{\prime}(1) / b}, \sqrt{2 C^{\prime}(0) / b}$ and $-\sqrt{2 C^{\prime}(0) / b},-\sqrt{2 C^{\prime}(1) / b}$. The interior solution (51) determines the map $r$ only in the region between the relevant lines as in (36), or the corresponding map in deviations from the steady state $x$ in (37). The implicitly defined part of $r$ in (37) is the function $r\left(x_{t}\right)$ that solves

$$
E S\left(x_{t}, r\left(x_{t}\right)\right)=q_{t}^{*} b\left(r\left(x_{t}\right)-x_{t}\right)+b x_{t}-B r\left(x_{t}\right)=0
$$

with

$$
q_{t}^{*}=q\left(x_{t}, x_{t+1}\right)=\left(C^{\prime}\right)^{-1}\left(\frac{b}{2}\left(x_{t+1}-x_{t}\right)^{2}\right) .
$$

Notice that, because $C^{\prime}(q)$ is increasing, $\left(C^{\prime}\right)^{-1}$ is always a well defined function and is itself increasing. We claim that the map $r$ has exactly the same properties $(\mathrm{a}-\mathrm{d})$ as the map $g$ in the proof of Theorem 4 . From property $(c)$ it follows that when $b / B<1,-1<g^{\prime}(x)<1$, so that the map is a contraction and all orbits converge to the steady-state $p^{*}$. From properties $(a-d)$ and the proof of Theorem 4 it follows that when $b / B>1$ the steady state is locally unstable and a unique globally stable 2-cycle exists.

We conclude the proof by showing that properties $(\mathrm{a}-\mathrm{d})$ hold in this case.

(a) From (52) it follows immediately that if $E S(x, r(x))=0$, then $E S(-x,-r(x))=0$. Hence, $r(-x)=-r(x)$. (b) We show that $y=r(x)$ has only one positive intersection $a$ with the line $y=-x$. This follows from a change in variables $z=x+r(x)$ and $w=r(x)-x$ as in the proof of Theorem 4 . In this case the corresponding system (42) has a solution only when $b / B>1$.

(c) Obviously when the map in (37) is explicitly defined, $r^{\prime}(x)=0$ or $r^{\prime}(x)=-b / B$. To compute $r^{\prime}(x)$ when it is implicitly defined one can use the implicit function theorem and obtain:

$$
r^{\prime}\left(x_{t}\right)=-\frac{\partial E S / \partial x_{t}}{\partial E S / \partial x_{t+1}}=\frac{q^{\prime}\left(x_{t}, x_{t+1}\right)\left(x_{t}-x_{t+1}\right)^{2}+q\left(x_{t}, x_{t+1}\right)-1}{q^{\prime}\left(x_{t}, x_{t+1}\right)\left(x_{t}-x_{t+1}\right)^{2}+q\left(x_{t}, x_{t+1}\right)+\frac{B}{b}} .
$$

Because both $q$ and $q^{\prime}$ are always positive, from the expression above it follows that $r^{\prime}\left(x_{t}\right) \in[-b / B, 1)$. Notice that $q^{\prime}$ is positive iff $C^{\prime}(q)$ is convex.

(d) Consider the proof of property $(d)$ in Theorem 4 . One can prove here that the same statement holds by replacing (52) with (38) and (53) with (43).

Proof of Theorem 13: Comparing (34) and (12), we have to show that there exists a non-decreasing, convex, information gathering cost function $C(q)$, such that the optimal solution of the representative agent problem (32) is given by:

$$
q_{t}^{*}=n_{1, t}=\frac{1+\tanh \left\{\frac{\beta}{2}\left[\frac{b}{2}\left(p_{t+1}-p_{t}\right)^{2}-C\right]\right\}}{2} .
$$


From the first order condition in (33) for an arbitrary cost function we obtain:

$$
q_{t}^{*}=\left(C^{\prime}\right)^{-1}\left(\frac{b}{2}\left(p_{t+1}-p_{t}\right)^{2}\right) .
$$

Combining (54) and (55) it follows that:

$$
\left(\frac{b}{2}\left(p_{t+1}-p_{t}\right)^{2}\right)=C^{\prime}\left(\frac{1+\tanh \left(\beta\left[\frac{b}{2}\left(p_{t+1}-p_{t}\right)^{2}-C\right]\right)}{2}\right) .
$$

In terms of the variable $z=b / 2\left(p_{t+1}-p_{t}\right)^{2} \geq 0$, (56) becomes:

$$
z=C^{\prime}\left(\frac{1+\tanh \left\{\frac{\beta}{2}[z-C]\right\}}{2}\right) \text {. }
$$

The change of variable $t=(1+\tanh (\beta(z-C) / 2)) / 2$ gives an ordinary differential equation:

$$
\frac{2 \tanh ^{-1}(2 t-1)}{\beta}+C=C^{\prime}(t)
$$

whose solution is the cost function $C(q)$ we are looking for. The restriction $z \geq 0$ implies $t \geq$ $(1+\tanh (-\beta C / 2)) / 2=t_{0}$. In integral form, $C(q)$ is given by:

$$
C(q)=\int_{t_{0}}^{q}\left(\frac{2 \tanh ^{-1}(2 t-1)}{\beta}+C\right) d t+C_{0}, \quad q \in\left(t_{0}, 1\right] .
$$

Notice that $t_{0}=\frac{1+m^{*}}{2}, C\left(t_{0}\right)=C_{0}$ and $C^{\prime}\left(t_{0}\right)=0$. This guarantees that when $p_{t}=p_{t+1}=p^{*}$ the solution of (55) is $q^{*}=\left(1+m^{*}\right) / 2$, the minimum fraction of rational agents at the fixed point in the heterogeneous agent model. When $q<t_{0}$ one can define without loss of generality, $C(q)=C_{0}$, and take the integration constant $C_{0}=0$. As a result one gets

$$
C(q)= \begin{cases}\int_{t_{0}}^{q}\left(\frac{2 \tanh ^{-1}(2 t-1)}{\beta}+C\right) d t & q \in\left(t_{0}, 1\right] \\ 0 & q \in\left[0, t_{0}\right] .\end{cases}
$$

By construction, the function $C(q)$ above has derivative $C^{\prime}(q)=0$ when $q \leq t_{0}$, and $C^{\prime}(q)$ as in (57) when $q>t_{0}$. Using this plus the fact that the function $(\tanh )^{-1}(2 t-1)$ is positive and increasing when $t \geq t_{0}$, it follows that the map $C(q)$ is non-decreasing and convex.

\section{References}

Anderson, S., A. de Palma, and J. Thisse (1993), Discrete Choice Theory of Product Differentiation, Cambridge: MIT Press.

Arthur, W. B. (1994), "Complexity in economic theory. Inductive reasoning and bounded rationality," American Economic Review, AEA Papers and Proceedings, 84, 406-11.

Brock, W. A., and C. H. Hommes (1997), “A rational route to randomness,” Econometrica 65, 1059-95.

Brock, W. A., and C. H. Hommes (1998), "Heterogeneous beliefs and routes to chaos in a simple asset pricing model," Journal of Economic Dynamics and Control 22, 1235-74.

Bullard, J. (1994), “Learning equilibria,” Journal of Economic Theory 64, 468-85.

Camerer, C. F., T.-H., Ho, and J. K. Chong (2002), "Sophisticated EWA Learning and Strategic Teaching in Repeated Games," Journal of Economic Theory 104, 137-88.

Diks, C. G. H., and P. Dindo (2006), “A behavioral model for participation games with negative feedback," CeNDEF Working Paper 06-10, University of Amsterdam, Amsterdam, the Netherlands. 
Dindo, P., and J. Tuinstra, (2006), "Coordination and competition in participation games," CeNDEF Working Paper, University of Amsterdam, the Netherlands.

Droste, E., Hommes, C., and J. Tuinstra (2002), "Endogenous fluctuations under evolutionary pressure in Cournot competition," Games and Economic Behavior 40, 232-69.

Dudek, M. K. (2004), "Expectation formation and endogenous fluctuations in aggregate demand," mimeo, Warsaw School of Economics, Warsaw, Poland.

Evans, G. W., and G., Ramey (1992), "Expectation calculation and macroeconomic dynamics," American Economic Review 82, 207-24.

de Fontnouvelle, P. (2000), "Information dynamics in financial markets," Macroeconomic Dynamics 4, $139-69$.

Goeree, J. K., and C. H. Hommes (2000), “Heterogeneous beliefs and the non-linear cobweb model,” Journal of Economic Dynamics and Control 24, 761-98.

Grandmont, J.-M. (1982), “Temporary general equilibrium theory,” K. J. Arrow, and M. D. Intriligator, eds, Handbook of Mathematical Economics, vol. 2, 879-922. Amsterdam: North-Holland.

Grandmont, J.-M. (1985), “On endogenous competitive business cycles,” Econometrica 53, 995-1046.

Grandmont, J.-M. (1998), "Expectation formation and stability in large socio-economic systems,” Econometrica 66, 741-81.

Hommes, C. H. (2006), “Heterogeneous agent models in economics and finance,” L. Tesfatsion, and K. L. Judd, eds, Handbook of Computational Economics, vol. 2, 1109-86, Elsevier Science.

Hommes, C. H. and G. Sorger (1998), “Consistent expectations equilibria,” Macroeconomic Dynamics 2, $287-321$.

Kirman, A. (1992), “Whom or what does the representative individual represent?” Journal of Economic Perspectives 6, 117-36.

Le Baron, B. (2006), “Agent based model in finance,” L. Tesfatsion, and K. L. Judd, eds, Handbook of Computational Economics, vol. 2, 1187-234. Elsevier: North Holland.

McKelvey, R. D., and T. R. Palfrey (1995), "Quantal response equilibria in normal form games," Games and Economic Behavior 10, 6-38.

McKelvey, R. D., and T. R. Palfrey (1998), “Quantal response equilibria for extensive form games,” Experimental Economics 1, 9-41.

Radner, R. (1982), “ Equilibrium under uncertainty,” K. J. Arrow, and M. D. Intriligator, eds, Handbook of Mathematical Economics, vol. 2, 923-1006. Amsterdam: North-Holland.

Schönhofer, M. (1999), “Chaotic learning equilibria,” Journal of Economic Theory 89, 1-20.

Simon, H. A. (1955), “A behavioral model of rational choice,” Quarterly Journal of Economics 69, 99-118.

Simon, H. A. (1957), Models of Man, New York, NY: Wiley.

Tuinstra, J. (2003), "Beliefs equilibria in an overlapping generations model," Journal of Economic Behavior and Organization 50, 145-64. 